\title{
Eta Carinae across the 2003.5 Minimum: Analysis in the visible and near infrared spectral region ${ }^{1}$
}

\author{
K. E. Nielsen ${ }^{2,3}$, G. Vieira Kober ${ }^{2,3}$, K. Weis ${ }^{4}$, T. R. Gull ${ }^{3}$, O. Stahl ${ }^{5}$, D. J. Bomans ${ }^{4}$ \\ nielsen@milkyway.gsfc.nasa.gov
}

\begin{abstract}
We present analysis of the visible through near infrared spectrum of $\eta$ Car and its ejecta obtained during the " $\eta$ Car Campaign with the Ultraviolet Visual Echelle Spectrograph (UVES) at the ESO Very Large Telescope (VLT)". This is a part of larger effort to present a complete $\eta$ Car spectrum, and extends the previously presented analyses with the Hubble Space Telescope/Space Telescope Imaging Spectrograph (HST/STIS) in the UV (1240-3159 $\AA$ ) to 10,430 . The spectrum in the mid and near UV is characterized by the ejecta absorption. At longer wavelengths, stellar wind features from the central source and narrow emission lines from the Weigelt condensations dominate the spectrum. However, narrow absorption lines from the circumstellar shells are present. This paper provides a description of the spectrum between 3060 and 10,430 $\AA$, including line identifications of the ejecta absorption spectrum, the emission spectrum from the Weigelt condensations and the P-Cygni stellar wind features. The high spectral resolving power of $V L T /$ UVES enables equivalent width measurements of atomic and molecular absorption lines for elements with no transitions at the shorter wavelengths. However, the ground based seeing and contributions of nebular scattered radiation prevent direct comparison of measured equivalent widths in the VLT/UVES and HST/STIS spectra. Fortunately, HST/STIS and $V L T /$ UVES have a small overlap in wavelength coverage which allows us to compare and adjust for the difference in scattered radiation entering the instruments' apertures. This paper provide a complete online $V L T / \mathrm{UVES}$ spectrum with line identifications and a spectral comparison between HST/STIS and VLT/UVES between 3060 and $3160 \AA$.
\end{abstract}

\footnotetext{
${ }^{2}$ Catholic University of America, Washington, DC 20064

${ }^{3}$ Astrophysics Science Division, Code 667, Goddard Space Flight Center, Greenbelt, MD 20771

${ }^{4}$ Astronomisches Institut, Ruhr-Universität Bochum, Universitätsstrasse 150, D-44780 Bochum, Germany

${ }^{5}$ Landessternwarte Heidelberg-Köningstuhl, D-69117 Heidelberg, Germany
} 
Subject headings: circumstellar matter - line identifications - stars: individual $(\eta \mathrm{Car})$

\section{Introduction}

Eta Carinae ( $\eta$ Car) is a massive Luminous Blue Variable (LBV, Conti 1984), that enriches the interstellar medium through major mass eruptions as it transitions from hydrogen burning to helium core nuclear energy production (Humphreys \& Davidson 1994). During the LBV phase, the star, for a relatively brief time interval, brightens as a consequence of a major lift-off of the stellar atmosphere's outer layers. A $60 M_{\odot}$ star may lose up to 10 $M_{\odot}$ while on the main sequence, but its pre-supernova mass is thought to be about $20 M_{\odot}$ (Smith \& Owocki 2006). The LBV phase is likely an evolutionary stage which most massive stars encounter during their lifetime, before exploding as a supernova (Meynet \& Maeder 2003). Growing evidence supports that some core-collapse supernovae have LBV progenitors (e.g. Smith et al. 2007; Gal-Yam et al. 2007), enhancing the cosmological importance of the LBVs.

Eta Car has a past with LBV characteristic events. The ejecta, formed during the Great Eruption in the 1840s and the less dramatic event in the 1890s, modifies $\eta$ Car's spectrum. In the 1840s more than $12 M_{\odot}$ of material was ejected (Smith et al. 2003b) and formed the bipolar Homunculus with an intervening disk (Davidson et al. 2001). In the 1890s an ionized bipolar structure, the Little Homunculus, formed interior to the Homunculus with a total mass of $\sim 0.5 M_{\odot}$ (Ishibashi et al. 2003). The Homunculus reflection nebula covered approximately $10^{\prime \prime} \times 18^{\prime \prime}$ on the sky in 2000 (Morse et al. 2001; Davidson et al. 2001), while the Little Homunculus is about $4^{\prime \prime}$ in extent (Ishibashi et al. 2003). The Weigelt condensations (Weigelt \& Ebersberger 1986) are bright emission structures currently located 0".2 to 0".3 from the stellar system. They date back to the event in the 1890 s based upon proper motion measurements (Davidson et al. 1995; Smith et al. 2004). A peculiar ionized region, the Strontium filament, lies within the disk (Zethson et al. 2001) and is excited by H I Balmer continuum radiation filtered by singly ionized iron (Hartman et al. 2004). The Strontium filament's spectrum shows lines of allowed and forbidden Sr II lines but is dominated by Ti II, Ni II and Fe I. Neither H I nor He I, and only weak O I and C I emissions are observed. Outside the Homunculus, a larger nebula of thin filaments, the outer ejecta, is observed. The outer ejecta is expanding with velocities up to $2000 \mathrm{~km} \mathrm{~s}^{-1}$ (Weis et al. 2004) with a

\footnotetext{
${ }^{1}$ Based on observations collected at the European Southern Observatory, Paranal, Chile during programs: 070.D-0607, 071.D-0168, 072.D-0524, 074.D-0141, 077.D-0618, 380.D-0036 and HST GO program 9973
} 
total mass of $\sim 0.5 M_{\odot}$ (Weis \& Duschl 1999).

Studies of the nebular emission revealed a nitrogen-enhanced but carbon- and oxygenstarved system (Davidson et al. 1986; Dufour et al. 1997). Smith \& Morse (2004) observed depleted oxygen abundances but enhanced nitrogen in the ejecta located immediately outside the Homunculus, while the ejecta at larger distance has solar composition. Smith \& Morse concluded that the ejecta further away from the central object was thrown long before the creation of the Homunculus and was less CNO processed. Verner et al. (2005b) suggested that oxygen and carbon are depleted by two orders of magnitude in the Weigelt condensations. The massive star that ejected this nitrogen-rich material was in its hydrogen burning evolutionary stage. Massive stars $\left(>40 M_{\odot}\right)$ are shown to have enhancements of nitrogen at the expense of carbon and oxygen. This is an abundance characteristic more pronounced due to mixing caused by stellar rotation (Meynet \& Maeder 2000, 2003). The remaining oxygen is tied up in corundum and simple molecules, leaving much of the metals in their gaseous atomic phase (Chesneau et al. 2005). The dust in the $\eta$ Car system has been discussed by Smith et al. (2003b); Smith (2002, and references there in), who observed a dust torus in the central parts of the system and a dusty Homunculus. However, the evidence is sparse as to if carbon is bound in the dust in the system. Furthermore, the properties of dust formation in a nitrogen-rich chemistry is rarely addressed.

Eta Car has a spectroscopic period, initially discovered through variations in He I $\lambda 10830$ integrated flux (Damineli 1996, and references within). The variation in excitation is accompanied by eclipse-like minima observed with $U B V$ and $B V R$ photometry (van Genderen et al. 2003), in near infrared $J H K L$ photometry (Whitelock et al. 2004) and in X-ray brightness (Ishibashi et al. 1999; Corcoran 2005). Corcoran (2005, and references therein) fine-tuned $\eta$ Car's period to $2024 \pm 2$ d (5.54 year) based on variability in the X-ray emission. Recently, Damineli et al. (2008) showed that the visible spectrophotometry and X-ray variability give consistent results. Most, if not all, observations can be explained by the presence of a companion star in a highly eccentric orbital with a 5.54 year period. A discussion about the binarity of the system can be found in several papers including Nielsen et al. (2007b), where the binary solution is compared to a single star model that attributes the spectroscopic variability from a latitudinal dependent stellar wind (Smith et al. 2003a). We adopted the binary solution for the spectral analysis presented in this paper, where the periastron passage represents the system's spectroscopic low-state and the time outside the periastron passage is the spectroscopic high-state.

Much attention has been devoted to find direct spectral evidence for the companion star $(\eta$ Car B) or signatures from the interacting winds. The X-radiation was demonstrated to originate from the wind-wind interface region (Ishibashi et al. 1999; Corcoran et al. 2001) and was modeled as interaction between a primary star ( $\eta$ Car A) with a dense wind $\left(\dot{M} \sim 2.5 \times 10^{-4} M_{\odot}\right.$ year $\left.^{-1}, v_{\infty}=500 \mathrm{~km} \mathrm{~s}^{-1}\right)$ and a fast companion wind $\left(\dot{M} \sim 10^{-5}\right.$ 
$M_{\odot}$ year $^{-1}, v_{\infty}=3000 \mathrm{~km} \mathrm{~s}^{-1}$; Pittard \& Corcoran 2002). Steiner \& Damineli (2004); Stahl et al. (2005) detected He II in the spectrum of $\eta$ Car indicating the presence of a hot companion. The He II emission was demonstrated by Martin et al. (2006) to originate from the wind-wind interface where highly excited lines normally not observed in the wind spectrum of a 15,000 K star could form. Nielsen et al. (2007a) traced, with HST/STIS spectra, He I lines formed in the wind-wind interface over the 5.54 year spectroscopic period to further analyze the ionization structure of the wind and derive $\eta$ Car B's orbital parameters. The spectrum of the Weigelt condensations during the broad maximum is consistent with the presence of a 37,000 K object, implying a companion star of O or WR-type (Verner et al. 2005b). Iping et al. (2005) found evidence for a hot companion by the far-UV flux behavior, but could not characterize it any further as the far-UV spectrum is modified by $\eta$ Car A's extended wind and the circumstellar material. All evidence favor a scenario where $\eta$ Car is a massive binary.

VLTI/VINCI interferometry (van Boekel et al. 2003) infered that $\eta$ Car A is a prolate spheroid, extending along the polar axis of the Homunculus, consistent with a polar wind with variable velocity during the spectroscopic high-state that decreased during the 1998.0 low-state (Smith et al. 2003a). The results from van Boekel et al. agree well with more recent VLTI/AMBER interferometry, using visibility functions of He I at $2.059 \mu \mathrm{m}$ and H I $\operatorname{Br} \gamma$ (Weigelt et al. 2007).

Multiple HST programs monitored the spectroscopic variations in the wavelength range 1175-10,300 $\AA$ from 1998.0 to 2004.3. The HST/STIS data provides invaluable information about the character and geometry of $\eta$ Car and its ejecta, including the geometry of the Homunculus (Davidson et al. 2001) and the Little Homunculus (Ishibashi et al. 2003). The initial spectral analysis using data obtained with HST/STIS resulted in atlases for the complete medium (R 30,000, 1240-2360 $\AA$; Nielsen et al. 2005) and high (R 110,000, 2380-3159 ^; Gull et al. 2006) resolution STIS MAMA wavelength region in the sightline towards the star. Spectral atlases, using the HST/STIS CCD spectrum (R 8000), for the Strontium filament and the Weigelt condensations are found in Hartman et al. (2004) and Zethson (2001), respectively. The Weigelt D UV spectrum was, with limited wavelength coverage, investigated with high resolution STIS MAMA spectra (Nielsen et al. 2007a). These atlases serve as a platform for further studies of $\eta$ Car's ejecta and circumstellar material around other objects such as gamma ray burst progenitors (Prochaska et al. 2006; Vreeswijk et al. 2007) or other LBVs (Nielsen et al., in prep).

Weis et al. (2005); Bomans et al. (2005) did the initial analysis of $\eta$ Car's VLT/UVES spectra and noted ejecta absorption lines including the Na I, K I and Ca II doublets. The VLT/UVES data were obtained during the " $\eta$ Car Campaign with UVES at the ESO VLT" which is a long term project for spectroscopic monitoring of $\eta$ Car with VLT/UVES in two slit positions. The first slit position is centered on the star itself and provided the data used 
by Weis et al. (2005); Bomans et al. (2005) and is the base for the investigation presented in this paper. The second slit position is offset towards the south-east lobe of the Homunculus nebula. Stahl et al. (2005) used this slit position in their analysis of He II $\lambda 4686$ in the scattered spectrum of the central source.

$V L T /$ UVES provides the necessary spectral resolving power to for spectral investigations of the ejecta at longer wavelengths. The extended wavelength coverage includes species with transitions outside the $H S T$ /STIS echelle spectral range, such as Sr II, Sc II, NH and $\mathrm{CH}^{+}$. However, the VLT/UVES spectrum is not easily compared to the HST/STIS spectra, because of the added complication of ground-based seeing coupled with nebular emission and spatially extended wind features from $\eta$ Car A and B that enter the larger aperture.

This paper presents the UVES spectrum between 3060 and 10,430 $\AA$, with line identifications of the nebular emission features, the P-Cygni wind lines and the ejecta absorption. The paper provides the necessary tools for further investigations of $\eta$ Car's spectrum, including an atlas with line identifications in addition to an analysis of the scattered contribution to the UVES continuum. Section 2 presents the observational data used in the analysis. Section 3 discusses the spectra of the Weigelt condensations, the circumstellar material and the stellar wind. In Section 4 we compare the column density measurements in the $-513 \mathrm{~km} \mathrm{~s}^{-1}$ absorption component to understand the scattered light properties that strongly influence the VLT/UVES spectrum. The wavelength overlap between VLT/UVES and HST/STIS provide the means to investigate the UVES spectrum with respect to the scatter radiation from the central source and is invaluable for further investigations of the UVES spectrum. We discuss the wavelength region between 3060 and $3159 \AA$, and derive a correction factor for the added scattered stellar radiation in the UVES spectrum. The correction factor will be used in an abundance analysis of the ejecta, including all observed species in absorption, that currently is being prepared by Kober et al. The online edition of this paper includes the entire VLT/UVES spectrum with over 1500 line identifications. All wavelengths throughout the paper are in vacuum.

\section{Observations}

Coordinated, highly complementary, observations were made with the HST/STIS and the VLT/UVES before, during, and after the spectroscopic low-state centered on 2003.5. The STIS spectra were obtained at critical intervals and cover the UV spectral range with high spatial and spectral resolving power. The STIS echelle observation, used in this analysis, was obtained in 2004 March with the E230H grating setting, providing a $0^{\prime \prime}$.070 spatial resolution with $0^{\prime \prime} 0145$ spatial sampling and a spectral resolving power of 110,000 in the wavelength region $2380-3159 \AA$. The area that is covered by the STIS slit changes as a 
Table 1. HST/STIS vs. VLT/UVES

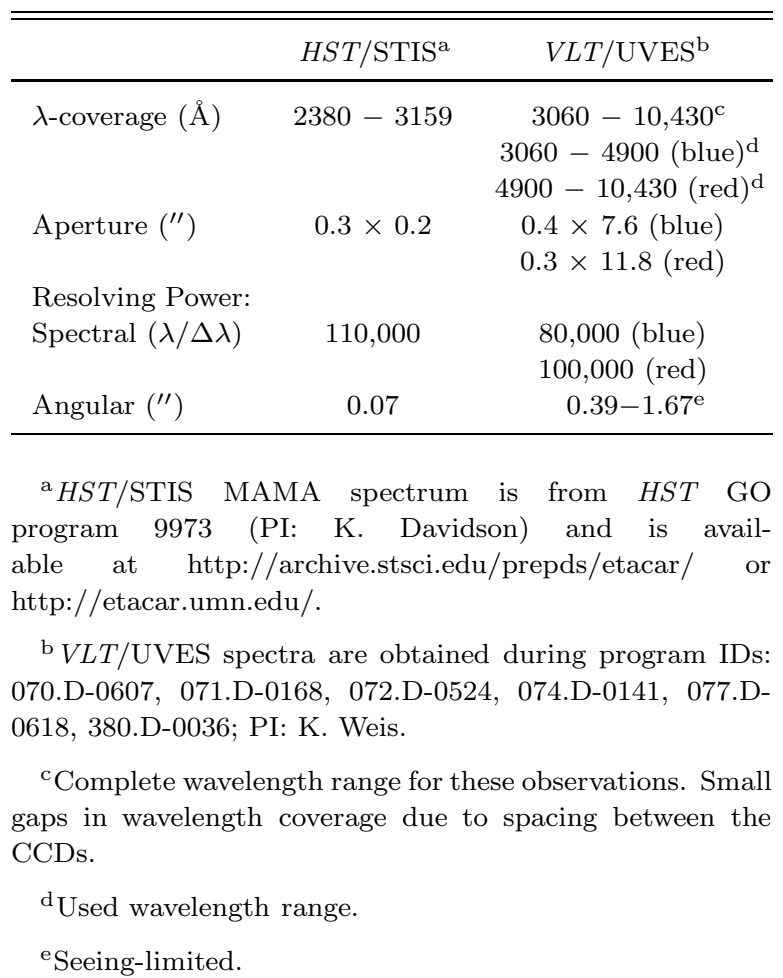


Table 2. Phase coverage with $V L T /$ UVES.

\begin{tabular}{llcc}
\hline \hline Instrument & Observation Date & JD $^{\mathrm{a}}$ & Phase, $\phi^{\mathrm{b}}$ \\
\hline VLT/UVES $^{\mathrm{c}}$ & 1999 Dec 21 & 1534 & 0.363 \\
& 2002 Dec 7 & 2616 & 0.897 \\
& 2002 Dec 12 & 2621 & 0.900 \\
& 2003 Feb 14 & 2685 & 0.931 \\
& 2003 May 29 & 2789 & 0.983 \\
& 2003 Jun 3 & 2794 & 0.985 \\
& 2003 Jul 5 & 2826 & 1.001 \\
& 2004 Feb 20 & 3056 & 1.115 \\
& 2005 Feb 12 & 3414 & 1.292 \\
& 2005 Mar 19 & 3449 & 1.309 \\
& 2006 Apr 9 & 3835 & 1.500 \\
& 2006 Jun 8 & 3895 & 1.529 \\
& & & \\
\hline
\end{tabular}

${ }^{\mathrm{a}}+2,450,000$

${ }^{\mathrm{b}}$ Phase relative to X-ray minimum 1997.9604, JD2,450,799.792+2024× ; Corcoran (2005).

${ }^{\mathrm{c}}$ The spectra used in this analysis is an extraction to include down to $15 \%$ of the flux from the central source.

${ }^{\mathrm{d}}$ The spectrum used in this analysis is a $0^{\prime \prime} 0145$ extraction centered on $\eta$ Car. 
function of the spacecraft roll-angle. For this particular observation, the HST orientation angle permitted use of the $00^{\prime \prime} 3 \times 00^{\prime \prime} 2$ aperture offset to include $\eta$ Car, Weigelt $\mathrm{B}$, and Weigelt D in a single observation. The spectrum, used in this analysis, is an extraction of seven $0^{\prime \prime} 0145$ wide high-resolution rows $\left(7 \times 0^{\prime \prime} \cdot 0145=00^{\prime \prime} 102\right)$ from the $H S T /$ STIS MAMA echelle image centered on $\eta$ Car. The 0 '.102 extraction minimizes contamination from the surrounding nebula and maximized the amount of information of the stellar spectrum. To avoid potential flux modulation, we used a spectral extraction that is slightly larger than what is needed based on the instrument's spatial resolving power. The HST/STIS data was acquired from the MAST data archive and reduced with a non-standard IDL reduction software from the STIS instrumental development team (Lindler 1999; Valenti et al. 2002).

The UVES spectra cover 3060-10,430 $\AA$, with small breaks due physical gaps between the CCD detectors, with 80,000 and 100,000 spectral resolving power in the blue $\left(\lambda_{c}=3460 \AA\right)$ and red $\left(\lambda_{c}=8600 \AA\right)$, respectively. Spectra were obtained frequently across the 2003.5 lowstate and with annual visits during the recovery period through 2006.4. All UVES high dispersion spectra were obtained with the same position angle $\left(\mathrm{PA}=160^{\circ}\right)$ as shown in Figure 1. While seeing was recorded by the observers to range between $0^{\prime \prime} .39$ to 1".67, the recorded line by line spectra have significantly larger FWHM, likely due to the extended geometry of $\eta$ Car. The recorded VLT/UVES spectra indicated significant larger stellar profiles, therefore, we used a slit extraction with spatial width to include down to $15 \%$ off-peak flux. For the spectrum obtained in 2002 December 12 we used a $33^{\prime \prime} 00$ and $2^{\prime \prime} .04$ extraction of the blue and red aperture, respectively. For observations obtained at the other phases an extraction as large as $>3$.' 75 was necessary to include the bulk of the flux from the star. The UVES data were reduced with pipeline methods. The resultant spatially resolved line-by-line spectra were produced by one of the authors (O. Stahl) with tailor made software to optimize the signal-to-noise in the overlap regions. The UVES spectra are not flux calibrated and comparisons between different spectra were done with the continuum normalized to unity.

The UVES and STIS characteristics are summarized in Table 1 and the observational phase coverage of $\eta$ Car's 5.54 year spectroscopic period is presented in Table 2. Figure 1 shows HST/Advanced Camera for Surveys optical images (GO program 9420; PI: K. Davidson) with the UVES apertures overlaid, including the portions of the apertures for which spectra were extracted for the 2002 December 12 observation.

We compare the VLT/UVES spectrum with the HST/STIS E230H in the overlap region between 3060 and $3159 \AA$ to gain insight of what is included in the UVES aperture. We extend the analysis to the entire covered UVES spectrum. The complete spectrum is presented as an atlas with line identifications in the online edition of the paper. 


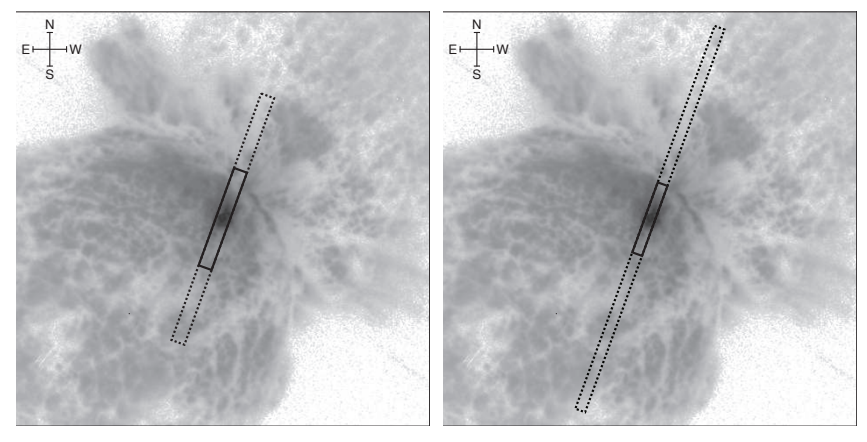

Fig. 1.- Image $\left(12^{\prime \prime} \times 12^{\prime \prime}\right)$ obtained with HST Advanced Camera for Surveys (GO program 9420; PI: K. Davidson). The UVES blue arm $\left(0^{\prime \prime} 4 \times 7^{\prime \prime} \cdot 6\right)$ and red arm $\left(0^{\prime \prime} 3 \times 11^{\prime \prime} .8\right)$ apertures are pictured (dashed) on the left and right images, respectively. In both figures the extracted parts of the apertures (solid) are depicted as used for the 2002 December 12 observation: $3{ }^{\prime \prime} 00$ in the blue arm and 2'.04 in the red arm. The cross-hair is $1^{\prime \prime}$ in both directions.

\section{The $\eta$ Car Spectrum}

The $\eta$ Car spectrum is modulated by the 5.54 year spectroscopic period. During the greater part of the period the system is influenced by radiation from the hotter $\eta$ Car B and is referred to as the spectroscopic high-state. The spectroscopic high-state is characterized by a higher level of ionization particularly in the Weigelt condensations and the circumstellar material. Across $\eta$ Car B's periastron passage, the system is dominated by radiation from $\eta$ Car A and exhibits lower ionization. The periastron passage lasts for a few months and is often called the spectroscopic minimum or low-state.

The spectra observed with VLT/UVES are seeing-limited and include extended stellar wind lines plus narrow line emission from the surrounding nebula, from the Weigelt condensations in particular. At wavelengths longward of $6250 \AA$, the Telluric $\mathrm{O}_{2}$ and $\mathrm{H}_{2} \mathrm{O}$ bands heavily contaminate the spectrum and significantly impair the analysis of the velocity variability and the changes in integrated flux of the stellar wind lines. In the following sections we discuss the origins of the identified lines in the spectrum, including the Weigelt condensations, the stellar wind and the circumstellar material. Figure 2 shows the current view of the system including how our sightline intersects the ejecta. Figure 3 shows an example of the UVES spectrum with identifications. It is a sample of the full spectrum that appears in the online edition of the paper. More information about the identified transitions in Figure 3 is presented in Table 3. The complete figure set, available online, is a comparison between a spectrum obtained during the spectroscopic high-state (2002 December 12, $\phi=0.90$ ) and one recorded during the low-state (2003 July 5). The two spectra are compared over the entire VLT/UVES wavelength region $(3060-10,430 \AA)$ 
Table 3. Identifications in the spectral interval 3160-3182 A.

\begin{tabular}{|c|c|c|c|c|c|c|c|}
\hline $\begin{array}{l}\lambda_{\mathrm{obs}^{\mathrm{a}}} \\
(\AA)\end{array}$ & Line ID & $\begin{array}{c}\lambda_{\text {lab }} \mathrm{b} \\
(\AA)\end{array}$ & Comment & $\begin{array}{l}\text { Velocity }^{\mathrm{c}} \\
\left(\mathrm{km} \mathrm{s}^{-1}\right)\end{array}$ & $\begin{array}{c}\mathrm{E}_{\text {low }}{ }^{\mathrm{d}} \\
\left(\mathrm{cm}^{-1}\right)\end{array}$ & $\log g f$ & Reference $^{\mathrm{e}}$ \\
\hline 3162.37 & Fe II $\left(d^{7} a^{4} P_{3 / 2}-4 p z^{4} F_{3 / 2}\right)$ & 3162.89 & $\mathrm{em}$ & -49 & 13905 & -2.84 & R98a \\
\hline 3163.19 & Fe II $\left(4 s c^{2} G_{7 / 2}-4 p y^{2} G_{7 / 2}\right)$ & 3163.71 & $\mathrm{em}$ & -49 & 33501 & -1.18 & R98a \\
\hline 3163.49 & Fe II $\left(d^{7} a^{4} P_{5 / 2}-4 p z^{4} F_{5 / 2}\right)$ & 3164.01 & $\mathrm{em}$ & -49 & 13474 & -2.85 & R98a \\
\hline 3164.03 & Ti II $\left(d^{3} b^{4} F_{9 / 2}-4 p z^{4} D_{7 / 2}\right)$ & 3169.45 & cs & -513 & 1216 & -0.14 & P01b \\
\hline 3167.06 & Fe II $\left(4 s a^{4} P_{5 / 2}-4 p z^{4} D_{3 / 2}\right)$ & 3167.58 & $\mathrm{em}$ & -49 & 13474 & -3.08 & R98a \\
\hline 3167.91 & Ti II $\left(d^{3} b^{4} F_{9 / 2}-4 p z^{4} D_{7 / 2}\right)$ & 3169.45 & cs & -146 & 1216 & -0.14 & $\mathrm{P} 01 \mathrm{~b}$ \\
\hline 3168.25 & Fe II $\left(4 s b^{2} G_{7 / 2}-4 p z^{2} G_{7 / 2}\right)$ & 3168.77 & em & -49 & 30764 & -0.72 & R98a \\
\hline 3168.34 & Fe II $\left(4 s b^{4} D_{5 / 2}-4 p x^{4} D_{7 / 2}\right)$ & 3168.86 & $\mathrm{em}$ & -49 & 31388 & -2.66 & R98a \\
\hline 3168.69 & Fe II $\left(4 s b^{2} G_{7 / 2}-4 p z^{2} G_{7 / 2}\right)$ & 3168.77 & wind & -8 & 30764 & -0.72 & R98a \\
\hline 3168.78 & Fe II $\left(4 s b^{4} D_{5 / 2}-4 p x^{4} D_{7 / 2}\right)$ & 3168.86 & wind & -8 & 31388 & -2.66 & R98a \\
\hline 3169.37 & Ti II $\left(d^{3} b^{4} F_{9 / 2}-4 p z^{4} D_{7 / 2}\right)$ & 3169.45 & wind & -8 & 1216 & -0.14 & P01b \\
\hline 3170.74 & Fe II $\left(d^{7} a^{4} P_{3 / 2}-4 p z^{4} D_{1 / 2}\right)$ & 3171.26 & em & -49 & 13676 & -2.61 & R98a \\
\hline 3172.47 & Cr II $\left(4 s a^{2} P_{3 / 2}-4 p z^{2} P_{1 / 2}\right)$ & 3172.99 & $\mathrm{em}$ & -49 & 35356 & -0.53 & K88 \\
\hline 3175.78 & [Fe II] $\left(4 s a^{6} D_{9 / 2}-4 s b^{4} D_{7 / 2}\right)$ & 3176.30 & $\mathrm{em}$ & -49 & 0 & -8.57 & G62 \\
\hline 3177.93 & Fe II $\left(4 s b^{4} D_{7 / 2}-4 p x^{4} D_{7 / 2}\right)$ & 3178.45 & $\mathrm{em}$ & -49 & 31483 & -0.90 & R98a \\
\hline 3178.06 & Fe II $\left(4 s b^{4} D_{1 / 2}-4 p y^{4} D_{1 / 2}\right)$ & 3178.58 & $\mathrm{em}$ & -49 & 31368 & -2.92 & R98a \\
\hline 3179.73 & Ca II $\left(4 p^{2} P_{3 / 2}-4 d^{2} D_{5 / 2}\right)$ & 3180.25 & $\mathrm{em}$ & -49 & 25414 & 0.51 & $\mathrm{~B} 72$ \\
\hline 3179.90 & Fe II $\left(4 s c^{2} D_{5 / 2}-4 p y^{2} F_{7 / 2}\right)$ & 3180.42 & $\mathrm{em}$ & -49 & 38164 & -0.87 & R98a \\
\hline 3180.06 & Cr II $\left(d^{5} a^{4} G_{11 / 2}-4 p z^{4} F_{9 / 2}\right)$ & 3181.61 & $\mathrm{cs}$ & -146 & 20512 & -0.20 & N06 \\
\hline 3181.09 & Cr II $\left(d^{5} a^{4} G_{11 / 2}-4 p z^{4} F_{9 / 2}\right)$ & 3181.61 & em & -49 & 20512 & -0.20 & N06 \\
\hline
\end{tabular}

Note. - CS, EM, WIND denotes the nature of the spectral line in the atlas, accordingly. CS is for circumstellar absorption lines, EM is emission from Weigelt D and WIND is stellar wind emission. Non-referenced data are from R. Kurucz's Atomic Line Database (http://cfa-www.harvard.edu/amdata/ampdata/kurucz23/sekur.html). A portion of the online table is shown here for guidance regarding its content.

${ }^{a}$ Calculated observed vacuum wavelength based on the heliocentric velocity.

${ }^{\mathrm{b}}$ Vacuum rest wavelength.

${ }^{\mathrm{c}}$ Heliocentric velocity.

${ }^{\mathrm{d}}$ Energy for the transitions lower state.

e Reference for $\log g f$.

References. - B72 - Black et al. (1972), G62 - Garstang (1962), K88 - Kurucz (1988), N06 - Nilsson et al. (2006), P01b - Pickering et al. (2001), R98a - Raassen \& Uylings (1998) 


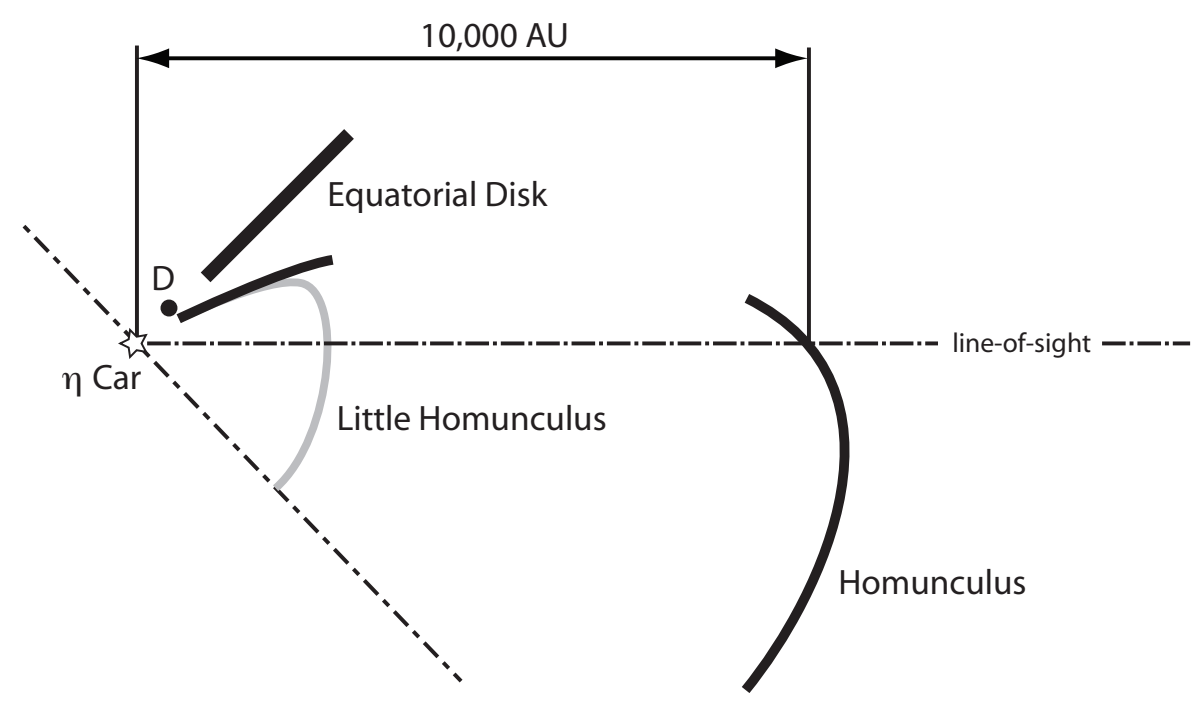

Fig. 2.- The current view of the $\eta$ Car system. The UV/visible spectrum is dominated by spectral lines primarily originating from the dense wind of $\eta$ Car A. Narrow emission lines are observed from Weigelt condensations, represented in the figure by Weigelt $\mathrm{D}$. The line-of-sight from $\eta$ Car intersects the ejecta, specifically the Little Homunculus and the Homunculus. Consequently, large parts of the observed spectrum are dominated by the ejecta absorptions.

\subsection{The Weigelt Condensations}

Zethson (2001) analyzed the Weigelt D emission spectrum for the wavelength region covered by $H S T /$ STIS CCD (1600-10,300 $\AA$, R 8,000). We used the work by Zethson as a guide to identify the narrow emission lines from the Weigelt condensations in the UVES spectrum. The majority of the narrow lines observed with HST/STIS are also observed in the UVES spectrum. However, weak lines are overpowered by the strong stellar radiation from $\eta$ Car A and B in the VLT/UVES spectrum. We note that the narrow component of the emission lines recorded with high-resolution $(R \sim 80,000) V L T /$ UVES are narrower than seen in the high-spatial, but lower spectral, resolution HST/STIS spectra.

The most prominent nebular features from the Weigelt condensations during $\eta$ Car's spectroscopic high-state are the H I Ly $\alpha$-pumped fluorescent lines in Fe II, especially Fe II $\lambda \lambda 2507,2509$ (Johansson \& Letokhov 2003). Transitions from higher ionization stages, such as $\mathrm{Fe}^{2+}, \mathrm{Fe}^{3+}, \mathrm{Ne}^{2+}$, are also identified in the spectrum. The radial velocities for the spectral lines in the spectrum of the Weigelt D condensation were determined by Nielsen et al. (2007b) to be $-47 \mathrm{~km} \mathrm{~s}^{-1}$ for the H I Ly $\alpha$-pumped lines and $-49 \mathrm{~km} \mathrm{~s}^{-1}$ for the allowed transitions. These derived radial velocities are consistent with previous results from Zethson (2001) and 
are used to identify the emission features in the VLT/UVES spectrum.

The H I Ly $\alpha$-pumped fluorescent lines and the forbidden lines of the highly ionized species are present during most of the 5.54 year cycle but disappear for several months during $\eta$ Car's low-state, coinciding with $\eta$ Car B's periastron passage. The spectral variability is attributed to the UV radiation field from the hot $\eta$ Car B (Verner et al. 2005b). During the periastron passage, the dense wind of $\eta$ Car A traps the FUV radiation from $\eta$ Car B in all directions. Figure 4 shows the differences in variability for the moderately ionized species, represented by [Fe II] $\lambda 4288$ (Fe I IP $7.9 \mathrm{eV}$ ), and the highly ionized [Ne III] $\lambda 3869$ (Ne II IP $40.9 \mathrm{eV}$ ) during the spectroscopic low-state and the recovery period. The highly ionized species are excited with radiation from $\eta$ Car B and, consequently, the most dramatic difference, as shown in the figure, is the absence of the [Ne III] $\lambda 3869$ at $-47 \mathrm{~km} \mathrm{~s}^{-1}$ beginning at phase $^{2}, \phi=1$, when the far-UV radiation from $\eta$ Car B is cut off. The [Fe II] $\lambda 4288$ depends upon mid-UV radiation and can be excited in the dense wind of $\eta$ Car A. The narrow nebular emission shows little correlation with $\eta$ Car's spectroscopic period. However, after the periastron passage $(\phi=1.115)$, a broad blue shifted emission feature appears at $-400 \mathrm{~km} \mathrm{~s}^{-1}$. By contrast the [Ne III] $\lambda 3869$ is absent at $\phi=1.0$ with only a weak narrow component at $\phi=1.115$. As the blue shifted component for [Fe II] $\lambda 4288$ fades, the [Ne III] $\lambda 3869$ grows stronger through $\phi=1.529$. The blue shifted condensation that is responsible for the emission component, is again being ionized by the far-UV radiation from $\eta$ Car B.

Zanella et al. (1984) noticed broad shoulders, blue-shifted up to $600 \mathrm{~km} \mathrm{~s}^{-1}$, of emission lines in their observed spectrum between 1981 and 1983. They concluded the components originated from a dense shells excited by the UV radiation from the hot core. The HST/STIS CCD spectrum, with 0"1 resolution, do not always show these broad shoulders since the STIS aperture, with orientation, samples a only thin slice of the wind structure. By comparing $H S T /$ STIS observations observed with different position angle and phase, we get indications of the origin of the broad emission components. A simple explanation for this emission is that it originates from the outer regions of the wind-wind interface. This would lead to a spatially resolved blue-shifted emission in highly excited lines, such as [Ne III] $\lambda 3869$, in a symmetric shell that is photo-ionized by $\eta$ Car B. In contrast, the less excited lines, for example [Fe II] $\lambda 4288$, are predominantly excited by the radiation from $\eta$ Car A.

\subsection{The Ejecta Absorption}

The ejecta, the Homunculus and the Little Homunculus, are oriented such that their walls intersects our sightline towards $\eta$ Car, leading to a major portion of the stellar radiation

\footnotetext{
${ }^{2}$ phase relative to X-ray minimum 1997.9604, JD2,450,799.792+2024× ; Corcoran (2005).
} 
being absorbed (see Figure 2). Consequently, $\eta$ Car's spectrum in line-of-sight is defined by the broad wind lines from $\eta$ Car A with nebular emission and circumstellar absorption superimposed. Gull et al. (2006) identified over 30 velocity components in the near-UV spectrum where two components, at -146 and $-513 \mathrm{~km} \mathrm{~s}^{-1}$, are isolated and distinguishable from the other. The Little Homunculus $\left(-146 \mathrm{~km} \mathrm{~s}^{-1}\right)$ is observed in absorption from metastable energy levels in mainly singly-ionized iron-group elements (Gull et al. 2005, 2006). Their energy levels are populated by photo-excitation and thermal collisions. The Homunculus $(-513$ $\mathrm{km} \mathrm{s}^{-1}$ ) spectrum is abundant in narrow absorption lines in neutral and singly-ionized metals plus several diatomic molecules (Verner et al. 2005a). The $-513 \mathrm{~km} \mathrm{~s}^{-1}$ spectrum is strong in the $\mathrm{H}_{2}$ Lyman bands located short-ward of $1650 \AA$ (Nielsen et al. 2005). These transitions are from states high up in the $\mathrm{H}_{2}$ energy level system not normally populated in a cold gas. With a strong background UV source, the $\mathrm{H}_{2}$ molecules can be excited followed by de-excitation to high vibrational and rotational states in the ground electronic state. The $\mathrm{H}_{2}$ lines vary in absorption depth with spectroscopic phase, which is in agreement with changing UV radiation from the binary system. Other molecules, for example, $\mathrm{CH}$ and $\mathrm{OH}$ are present in the HST/STIS spectrum, but no $\mathrm{CO}$ is observed. Additional molecules are observed in the VLT/UVES spectrum at longer wavelengths, such as $\mathrm{NH}$ and $\mathrm{CH}^{+}$

Gull et al. (2005) presented preliminary results for the abundance analysis of the $\eta$ Car ejecta, based on Fe II and Ti II column densities in the -146 and $-513 \mathrm{~km} \mathrm{~s}^{-1}$ velocity components, respectively. They derived column densities using a standard curve-of-growth method, where the results were used as input data for CLOUDY modeling. CLOUDY is a photo-ionization code where the energy level populations are calculated with a statistical equilibrium approach (Ferland et al. 1998). A Kurucz model atmosphere (Kurucz 1988) with $T_{\text {eff }}=15,000 \mathrm{~K}$ and $L=10^{40} \mathrm{ergs} \mathrm{s}^{-1}$, with carbon and oxygen 100 times depleted and nitrogen and helium 10 times enhanced, was used to mimic the radiative flux from $\eta$ Car A. All other metals were assumed to have solar abundances. The absorption at $-146 \mathrm{~km} \mathrm{~s}^{-1}$ was determined to originate in a gas located $1300 \mathrm{AU}$ from the radiative source, with a variable excitation temperature, $T_{e}=4700-5750 \mathrm{~K}$, dependent on the spectroscopic phase. The absorption at $-513 \mathrm{~km} \mathrm{~s}^{-1}$ is located $\sim 10,000 \mathrm{AU}$ from the stellar system and is much cooler, $T_{e}=760 \mathrm{~K}$. However, modeling of transitions in $\mathrm{CH}$ and $\mathrm{OH}$ yield a much lower temperature (30 K, Verner et al. 2005a), indicating formation of atomic and molecular absorption in two spatially separated regions. No measurable difference in velocity between the molecular and atomic absorption lines is observed. The absorption at $-513 \mathrm{~km} \mathrm{~s}^{-1}$ does not show any significant variability in atomic absorptions with phase. However, the strongly phase dependent $\mathrm{H}_{2}$ fluorescent spectrum nearly disappears during the periastron passage when the UV radiation from the central source is cut off. A more comprehensive study covering all observed species in the HST/STIS and the VLT/UVES spectra is underway (Kober et al. in prep). The results from the modeling by Gull et al. (2005) are summarized in Table 4. 
The larger VLT/UVES aperture covers the nebulosities surrounding $\eta$ Car, and includes radiation from the extended, dust scattered-stellar wind and narrow emission from the Weigelt condensations. Hence, the narrow line absorption as detected separately in the $H S T /$ STIS spectrum is included in the VLT/UVES spectra. However, many absorption lines at $-513 \mathrm{~km} \mathrm{~s}^{-1}$ and some at $-146 \mathrm{~km} \mathrm{~s}^{-1}$ are still identified and measured. VLT/UVES provides high dispersion spectra to $10,430 \AA$, and provides the opportunity to investigate additional atomic and molecular species which are not accessible with the high spectral resolution HST/STIS MAMA. The HST/STIS CCD spectrum with low spectral resolution, extends beyond 10,000 $\AA$ but do not show any signs of absorption features from the circumstellar shells. For the $-513 \mathrm{~km} \mathrm{~s}^{-1}$ associated with the Homunculus, UVES provides information about atomic absorption lines in Sr II, Sc II, K I, Ca I, Ca II, Na I, Cr I, Ti II, V II, Ni I, Fe I and molecular lines in $\mathrm{CH}, \mathrm{CH}^{+}$and $\mathrm{NH}$. The absorption spectrum is characterized by allowed transitions which are easier to interpret, regarding populations of the energy levels. Abundance analysis using absorption spectra provide more accurate results due to better known atomic/molecular data.

\subsection{Eta Car's Wind spectrum}

The spectral crowding of absorption features complicates observations of the wind lines in the UV. The ejecta absorption, especially, in the iron-group elements, blanket the spectrum and impairs the measurements of the wind lines and their time variability. At wavelengths longward of $3000 \AA$ the line density decreases and the wind lines, primarily from $\eta$ Car A, are observable. Most wind line profiles appear with a broad $\left( \pm 550 \mathrm{~km} \mathrm{~s}^{-1}\right)$ profile, often with P-Cygni absorption, plus a narrow $\left( \pm 15 \mathrm{~km} \mathrm{~s}^{-1}\right.$ centered at $\left.-47 \mathrm{~km} \mathrm{~s}^{-1}\right)$ emission component, originating from the Weigelt condensations. The presence of the narrow emission from the Weigelt condensations impairs the analysis of the velocity variation and the variability of the emission and absorption components of the wind lines. Many wind lines show significant variations in velocity and integrated flux over the 5.54 year spectroscopic period. The line variability is dependent on where the line is formed in the stellar wind. By investigating the phase dependance, information regarding the wind ionization structure and the influence of the companion star can be extracted. The wind spectrum between 3060 and 10,430 $\AA$ is dominated by the hydrogen Balmer and Paschen series. The hydrogen lines are predominantly formed in $\eta$ Car A's wind with terminal velocity of $\sim 550 \mathrm{~km} \mathrm{~s}^{-1}$. Their absorption profiles show small velocity variations, which likely are tied to the orbital motion of $\eta$ Car A. The weaker He I line profiles show more pronounced velocity variations as observed by Nielsen et al. (2007a). The He I lines originate from states high up in the energy level system $\left(<150,000 \mathrm{~cm}^{-1}\right)$ and are normally not present in a 15,000 K stellar spectrum. 
In the HST/STIS spectrum the He I lines show complex line profiles with multiple emission and absorption components. The lines were, based on their presence, velocity variation and line profile, determined to be formed in the wind-wind interface region between the two massive objects. With a lower spatial resolving power the multi-component structure is damped in the UVES spectrum leading to a smooth line profile. Nielsen et al. (2007a) used the HST/STIS observed P-Cygni absorption to derive a radial velocity curve. However, the relation between the derived velocity and the motion of the companion star is difficult to understand. The changes in the UVES observed line profiles and their variability over the spectroscopic period are shown in Figure 5 for the H I $\lambda 4103$ and He I $\lambda 7065$. Other species with strong prominent wind spectrum are Si II and Fe II with lines, due to their ionization potentials, formed primarily in the $\eta$ Car A's extended stellar wind.

\section{Column Density Measurements}

The abundance analysis is dependent on accurately measured elemental column densities. While the majority of the ejecta absorption lines useable for equivalent width measurements are in the HST/STIS spectrum (2380-3159 $\AA$ ), some species are uniquely observed in the VLT/UVES spectral region (3159-10,430 $\AA$ ). Meaningful comparisons of column densities require correcting the measure equivalent widths for all observational effects. The HST/STIS spectrum was obtained with near diffraction spatial resolution excluding emission from the surrounding nebula and the extended stellar wind. The seeing-limited spatial resolution of $V L T /$ UVES required a larger aperture that includes the emission from the Weigelt condensations and most of $\eta$ Car A's extended wind. To compare the observed spectra and equivalent widths for lines that are recorded with HST/STIS and HST/STIS, respectively, we used the small overlap in wavelength coverage between the two instruments (3060 to $3159 \AA)$.

The wavelength region 3060-3159 A has many Ti II and V II absorption lines. A subset

of the overlap wavelength region is presented in Figure 6. Ti II was used by Gull et al. (2005) to derive the physical parameters for the Homunculus. The column density is derived from a standard curve-of-growth, i.e. the equivalent width as function of the transition strength. To accurately calculate the column density for an energy state, the transitions from the from this energy level must be unblended, have accurately determined atomic data, and located in a wavelength region where the continuum can be properly set. At $3000 \AA$ the absorption line density is significantly lower, compared to the mid and far UV. The probability for blends decreases at longer wavelengths and makes the continuum placement easier, especially in the HST/STIS high-resolution spectrum that is unaffected by the strong emission from the surrounding nebula. The continuum placement for the UVES spectrum is affected by the 
scattered emission features from the nebula and, at longer wavelengths, the atmospheric absorption bands. In the wavelength region between 3060 and $3159 \AA$, the contribution from the atmospheric absorption is negligible and only a few lines are affected by the narrow emission from the Weigelt condensations. Our analysis does not include absorption lines that are obviously blended or where the continuum level could not be set with a satisfactory accuracy. Our confidence in setting the continuum is comparable for both the STIS and the UVES spectra in the wavelength region, 3060-3159 $\AA$, and is helped by the narrow width of the ejecta absorption lines $\left(b \sim 2.5 \mathrm{~km} \mathrm{~s}^{-1}\right)$. The overlap region allows us to make a direct comparison of the equivalent widths for a few Ti II lines measured in the UVES and STIS spectra. However, while using spectral lines in the overlap region to calculate a correction factor, additional Ti II lines at wavelengths outside the overlap region are included to derive and compare column densities. The Ti II transition probabilities are, with a few exceptions, derived from measured branching fractions and lifetimes (Pickering et al. 2001), complemented with data from Fuhr et al. (1988).

The absorption features recorded by HST/STIS are systematically deeper than identical features recorded with VLT/UVES. Hence, column densities derived from STIS spectra are larger than those from the UVES spectra. The Ti II $\lambda 3089$, shown in Figure 7 , is nearly saturated and falls close to the flat part of the curve-of-growth (see Figure 8). However, it does not reach zero flux. The line profiles in the UVES spectrum do not show the same absorption depth, indicating a difference in detected continuum for UVES and STIS. The altered flux level may be caused by additional radiation entering the line-of-sight or instrumental effects. The latter is a combination of inter-order radiation and instrumental resolving power. The inter-order characteristics of the echelle spectrograph with a cross-dispersion grating can be examined using spectral cross-cuts of the detector image. For both instruments the interorder contribution is small and corrected during the raw-data reduction process.

The spectral resolving power's effect on the spectral line profiles is investigated through convolving a saturated test spectral feature with a point-spread-function of desired line width to observe how the spectral line depth changes. We estimated that HST/STIS, with a spectral resolving power of $\mathrm{R} \sim 100,000$, changes the depth of the spectral feature by $5-10 \%$. This is in agreement with observations of the almost saturated Ti II $\lambda 3089$ in the HST/STIS spectrum. We conclude that the instrumental effects for STIS and UVES spectra are comparable in nature and do not explain the observed difference in absorption line depth.

Saturated absorption lines, not reaching zero flux level, are observed in, for example, Seyfert galaxy spectra (Kraemer et al. 2002), where the absorber does not cover the entire radiative source. A similar effect is seen even if the absorbing cloud does cover the entire radiative source, but there is an added contribution to the continuum through reflected or scattered radiation (see Figure 9). With a contribution to the continuum between the ab- 
sorber and the observer, the additional radiation will cancel parts of the observed absorption and a saturated line would not reach zero flux at any wavelength.

In the case of $\eta$ Car and its ejecta, the extended wind and the ejecta contribute scattered radiation to the stellar continuum in the $V L T /$ UVES. The $-513 \mathrm{~km} \mathrm{~s}^{-1}$ absorption is, however, determined to originate in the Homunculus that is located far from the radiative source. If the scattered radiation from the extended wind and the ejecta enters the light path before being absorbed in the Homunculus, the additional radiation will not alter the spectral line depth relative to the continuum.

The correction for the altered continuum is performed by estimating the offset by using saturated lines in the spectrum. If the radiative source is entirely covered by the absorbing gas, then a saturated line is expected to reach zero flux at its line center. A few Ti II lines in the spectral overlap region are close to the flat part of the curve-of-growth and, consequently, are assumed to be saturated. The Ti II $\lambda 3089$ is the best example of a saturated line in the overlap spectrum and is used to estimate the contribution of scattered radiation in the HST/STIS spectrum. The Ti II $\lambda 3089$ is the transition in the overlap region that is best suited to calculate the correction factor. However, a few other lines appear to be better candidates in the UVES spectrum. We have chosen Ti II $\lambda 3235$ to correct for scattered radiation in the UVES spectra. Ti II $\lambda 3235$ is clearly on the flat part of the curve-of-growth (see Figure 8) and has a well defined continuum. This spectral line is a slightly blended by additional velocity component from the same transition. The contribution from the blending components to the line depth is small and the contribution to the correction factor is assumed to be negligible. Only a few lines are close to being saturated in the observed spectral region. Rather than calculate an average contribution factor from all nearly saturated lines, which would add a systematic error to the analysis, we have chosen to use one single line to calculate the correction. By adjusting the continuum in the UVES and STIS spectra, it is possible to compare column densities measured in the different wavelength regions observed by different instruments. We calculate a correction factor for the scattered radiation by subtracting the flux between zero flux and the measured line depth for a saturated line, according to Equation 1.

$$
F_{c}=\frac{F}{F_{0}}-C_{f} .
$$

Where $F$ is the pre-normalization flux level, $F_{0}$ is the defined continuum level and $C_{f}$ is the correction factor. If the correction factor is applied to the column density measurement made in the UVES and STIS spectra, respectively, then the results are in good agreement. Figure 8 shows how we have applied the correction factor for spectral lines in the STIS and UVES region for the $4 s a^{4} F$ term in Ti II. The correction proved very effective in bringing 
the measured equivalent widths and derived column densities into agreement. Note, to accurately calculate the influence from scattered nebular light, saturated spectral lines must be used. Otherwise, it is difficult to distinguish between contribution from scattered light and lack of absorbing material in the gas. An analysis using V II at $-513 \mathrm{~km} \mathrm{~s}^{-1}$ produced similar results.

To correct for any wavelength dependence of the scattering properties in the nebula would require saturated spectral features throughout the spectral region. However, the correction is calculated based on the extracted spectrum in the blue spectral region. Most absorption features are located in the near-UV wavelength region. We have no independent measure for the correction factor in the red. For the absorption features at longer wavelength, the use of saturated Ti II lines only provides an estimate of the amount of scattered radiation entering the $V L T /$ UVES aperture.

The difference in scattered radiation observed in the STIS and UVES spectra, i.e. the contribution to the UVES continuum, varies with time. We find no correlation between added radiation with seeing conditions or zenith angle, but the continuum offset may change with $\eta$ Car's spectroscopic 5.54 year period, as shown in Figure 10. During the periastron passage the contribution from the scattered radiation appears to be greater compared with the rest of the spectroscopic period. This phase coincides with the phase $(\phi=1.0)$ when the radiation from the hot $\eta$ Car B is increasingly obscured and the wind opacity is higher, indicating a more efficient scatterer. While the Homunculus is time independent in atomic absorption, exciting radiation from the central source is dependent on the orbital position of $\eta$ Car B. Consequently, the variability in the continuum offset in the UVES spectrum is more likely associated with the scattered radiation from the extended primary wind than photons scattered by the Homunculus. If the radiation that modifies the continuum originates from the inner part of the nebula, then the absorber needs to be smaller than the radiative source. This is not fully in agreement with a Homunculus being a continuous absorber. For example, Smith (2006) depicted the emission from the Homunculus as a thin smooth skin in $\mathrm{H}_{2}$. A clumpy Homunculus is in many ways in better agreement with previous studies. The presence of molecules and singly ionized metals in the same gas observed at the same velocity, can be explained with temperature and density stratification along the Homunculus surface.

\section{Summary}

We have investigated the VLT/UVES spectrum between 3060 and 10,430 $\AA$ and identified most spectral features. The spectrum is a composite of spectral lines from $\eta$ Car A's 
wind, emission from the surrounding nebula and the Weigelt condensations in particular, and absorption in the Homunculus and Little Homunculus. The VLT/UVES spectra, from epochs $\phi=0.900$ and 1.001, are presented with over 1500 line identifications in the online edition of this paper.

The HST/STIS and VLT/UVES instruments have differences in spatial resolution and aperture size. We face the challenge to correct for the contribution of the nebular-scattered light entering the aperture. The scattered light will offset the continuum, which is a problem more pronounced for $V L T /$ UVES than for HST/STIS spectra due to seeing and the larger aperture size.

The HST/STIS spectrum is obtained with the $0 \prime 3 \times 0$ ' ${ }^{\prime} 2$ aperture, but to minimize the contribution from the surrounding nebula a 7 pixel extraction ( $\left.00^{\prime \prime} 102\right)$ was used in the analysis, providing critical separation of the stellar emission from dust scattered stellar radiation and nebular emission originating within the Homunculus. The UVES aperture is $0^{\prime \prime} 4 \times 7^{\prime \prime} \cdot 6$ for the blue spectrum, but we have used a subsection of the echelle image including flux from the central source down to a $15 \%$ level. The limited spatial resolution in the, seeing dependent, ground-based UV spectra explains why the curves-of-growth from these two data sets appear to show different results. The chaotic natures of the expanding ejecta within the Homunculus, the extended wind of the central source, and the many emission nebulosities lead to a velocity-smeared spectrum.

The additional flux in the HST/STIS can be explained either by the absorbing material in the Homunculus (at $-513 \mathrm{~km} \mathrm{~s}^{-1}$ ) being smaller than the radiative source in the center of the nebula, or by additional flux enters the aperture between the Homunculus and the observer. At the inferred distance of the Homunculus, $\sim 10,000 \mathrm{AU}$, the clumpy structure of the ejecta does not completely cover $\eta$ Car's extended wind, based upon the differences in ejecta absorption profiles, yet the clumps in the Homunculus must be significantly larger than the $0^{\prime \prime} 1$ that is covered by the HST/STIS aperture, but smaller than the portion covered by $V L T /$ UVES. The structure of the Homunculus has significance for its gas-to-dust ratio and implications for its mass. The current gas-to-dust ratio in the Homunculus is very uncertain and an improved value would increase the derived value of the Homunculus mass.

The amount of nebular/scattered light contribution, caused by scattered radiation, may change with orbital phase. This would make the correction even more problematic. Phase dependence of the scattered radiation would suggest changes with in dust properties in the centralregion, likely due to new grain formation/destruction or modification by the UV radiation. A possible drop in the scattered light occurs about 1.7 years $(\phi=0.3)$ after the spectroscopic low-states both in late 1999 and in 2005, however, the observed changes are on the same scale as the measurement errors.

We have corrected for the additional flux in the UVES spectrum with saturated Ti II lines in the blue and have shown that the correction puts the measurements from the 
$H S T /$ STIS and the VLT/UVES spectra in agreement. The correction of scattered radiation in UVES spectrum will be used to describe the abundance characteristics of $\eta$ Car's ejecta. That analysis is currently underway. 


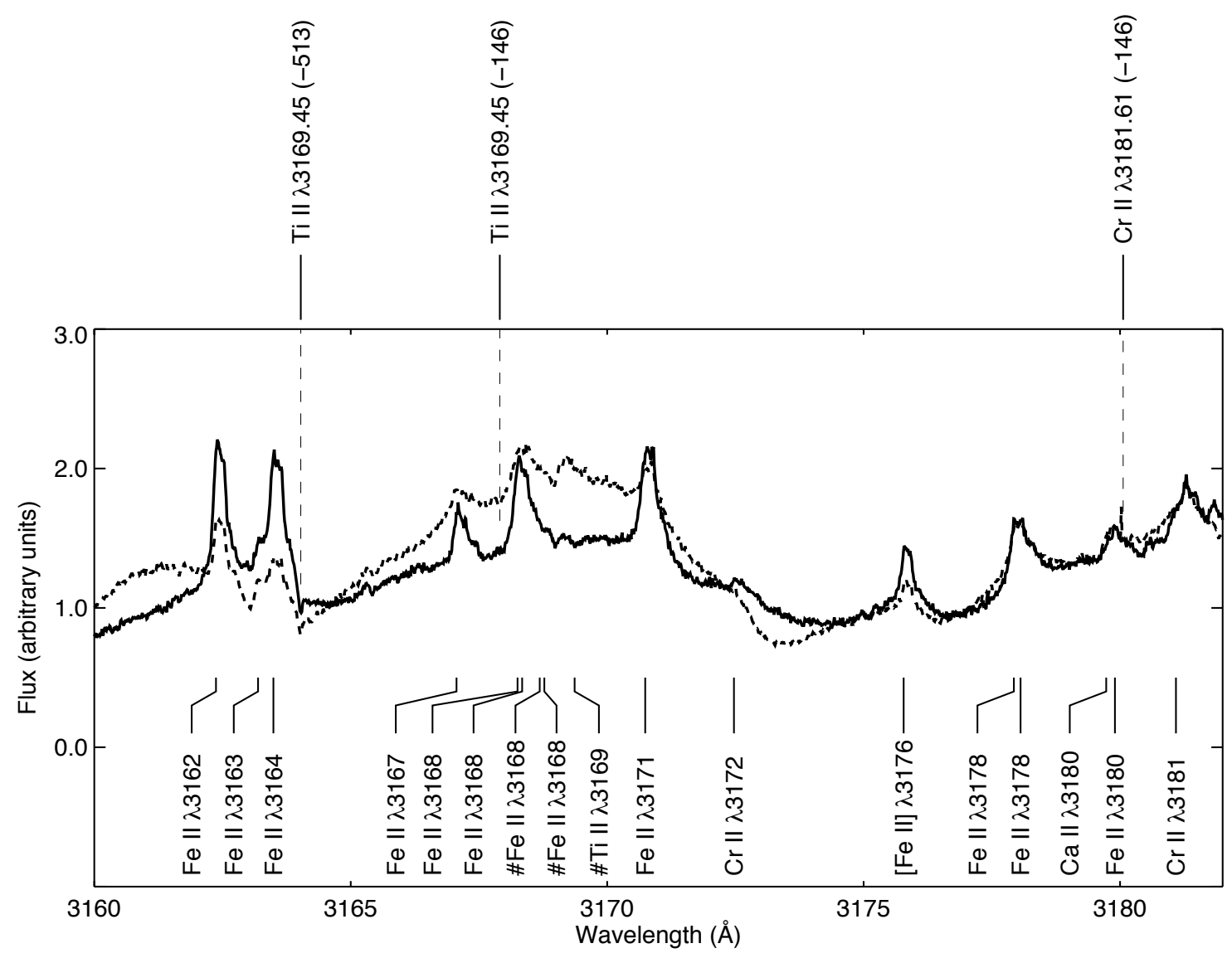

Fig. 3.- Extracted high-resolution spectrum obtained with VLT/UVES (R 80,000) of $\eta$ Car during the spectroscopic high-state (2002 December 12, solid) and spectroscopic lowstate (2003 July 5, dashed). Both spectra are dominated by the emissions from the Weigelt condensations and $\eta$ Car A's stellar wind lines. Narrow absorption features originating from the circumstellar material, are observed at -146 and $-513 \mathrm{~km} \mathrm{~s}^{-1}$. Identifications for the ejecta absorption lines are in the top of the figure with wavelength and radial velocity. Stellar wind lines and emission from the Weigelt condensations are identified in the bottom of the figure with wavelength. The wind lines are marked with \#. All identified lines are presented in Table 3. All wavelengths are in vacuum. 

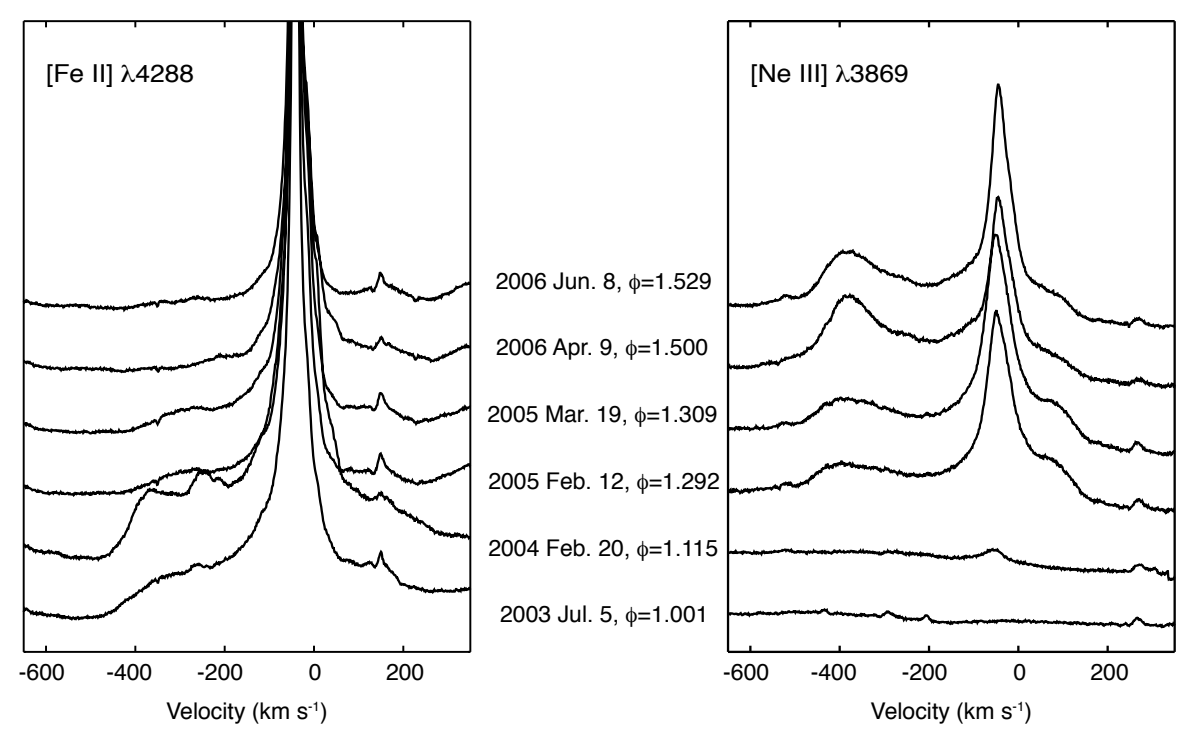

Fig. 4. - The recovery after the periastron passage of the emission lines from the Weigelt condensations and the surrounding nebula. The transitions from higher ionization states, represented by [NeIII] $\lambda 3869$, are absent during the spectroscopic low-state $(\phi=1.0)$ and reappear when the ionizing flux from $\eta$ Car B again penetrates $\eta$ Car A's wind. The spectral lines with lower ionization such as [FeII] $\lambda 4288$, are less dependent on the external UV radiation and show less change in strengths over the 5.54 year spectroscopic period. Note the emission feature at $-400 \mathrm{~km} \mathrm{~s}^{-1}$. This component is strongest for the low ionization features at $\phi=1.115$ while the high ionization lines peak at apastron $(\phi=1.500)$. The plots are offset vertically with increasing phase to show changes in the broad emission lines. 
Table 4. Ejecta parameter in part from Gull et al. (2005)

\begin{tabular}{lcc}
\hline \hline & $-513 \mathrm{~km} \mathrm{~s}^{-1}$ & $-146 \mathrm{~km} \mathrm{~s}^{-1}$ \\
\hline$T_{\max }(K)$ & 760 & $6400^{\mathrm{b}}$ \\
$T_{\min }(K)$ & $760^{\mathrm{c}}$ & $5000^{\mathrm{b}}$ \\
Ionization & Neutral, Singly & Singly \\
Distance $(\mathrm{AU})$ & 10,000 & 1300 \\
$\rho_{e}\left(\mathrm{~cm}^{-3}\right)$ & $10^{7}$ & $10^{7}-10^{8}$ \\
$b\left(\mathrm{~km} \mathrm{~s}^{-1}\right)$ & $2.1-2.5$ & $5.5-8.0$ \\
$v_{t}^{\mathrm{a}}\left(\mathrm{km} \mathrm{s}^{-1}\right)$ & $1.4-1.7$ & $3.8-5.6$ \\
\hline
\end{tabular}

aThe $b$-value is the Doppler width of the spectral line. In this definition of $b, v_{t}$ is the one-dimensional rms value for a gaussian spectrum of internal trubulent velocities, the full three dimensional turbulent velocity is thus $\sqrt{3} v_{t}$.

${ }^{\mathrm{b}}$ The temperatures have been revised with oscillator strengths from Raassen et al. (1998) yielding $T_{\min }=4700 \mathrm{~K}$ and $T_{\max }=5750 \mathrm{~K}$.

${ }^{\mathrm{c}}$ The temperature may change between the high and low states, but the difference is within the measurement error. The accuracy of the $T_{\min }$ is affected by blends due to additional velocity components that appear during the spectroscopic low-state. 

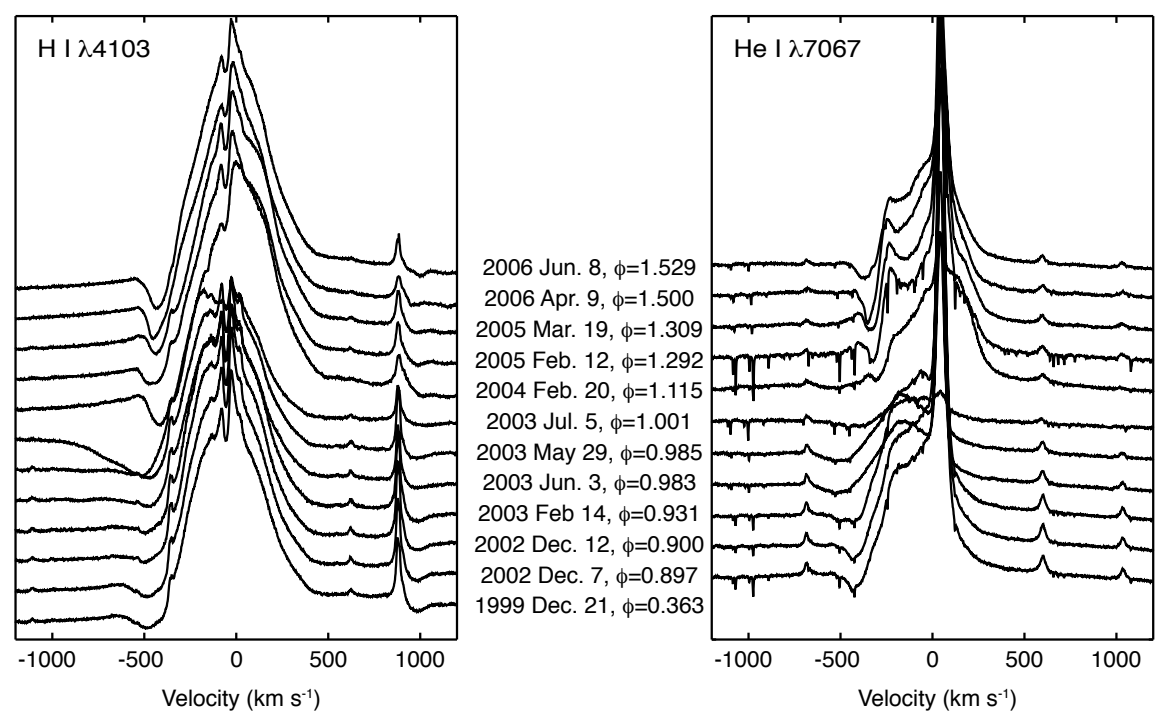

Fig. 5.- A comparison of the He I $\lambda 7067$ originating in the wind-wind interface region with H I $\lambda 4103(\mathrm{H} \delta)$ formed in $\eta$ Car A's wind. The Weigelt component dominates the emission of the He I line, but with an underlying complex structure formed in the two arms of the bow-shock. The absorption component of the P-Cygni shaped line show a very abrupt shift at periastron. A similar, but less dramatic, shift is observed in $\mathrm{H} \delta$, indicating the line is formed in a larger volume and further away from the radiative source. 


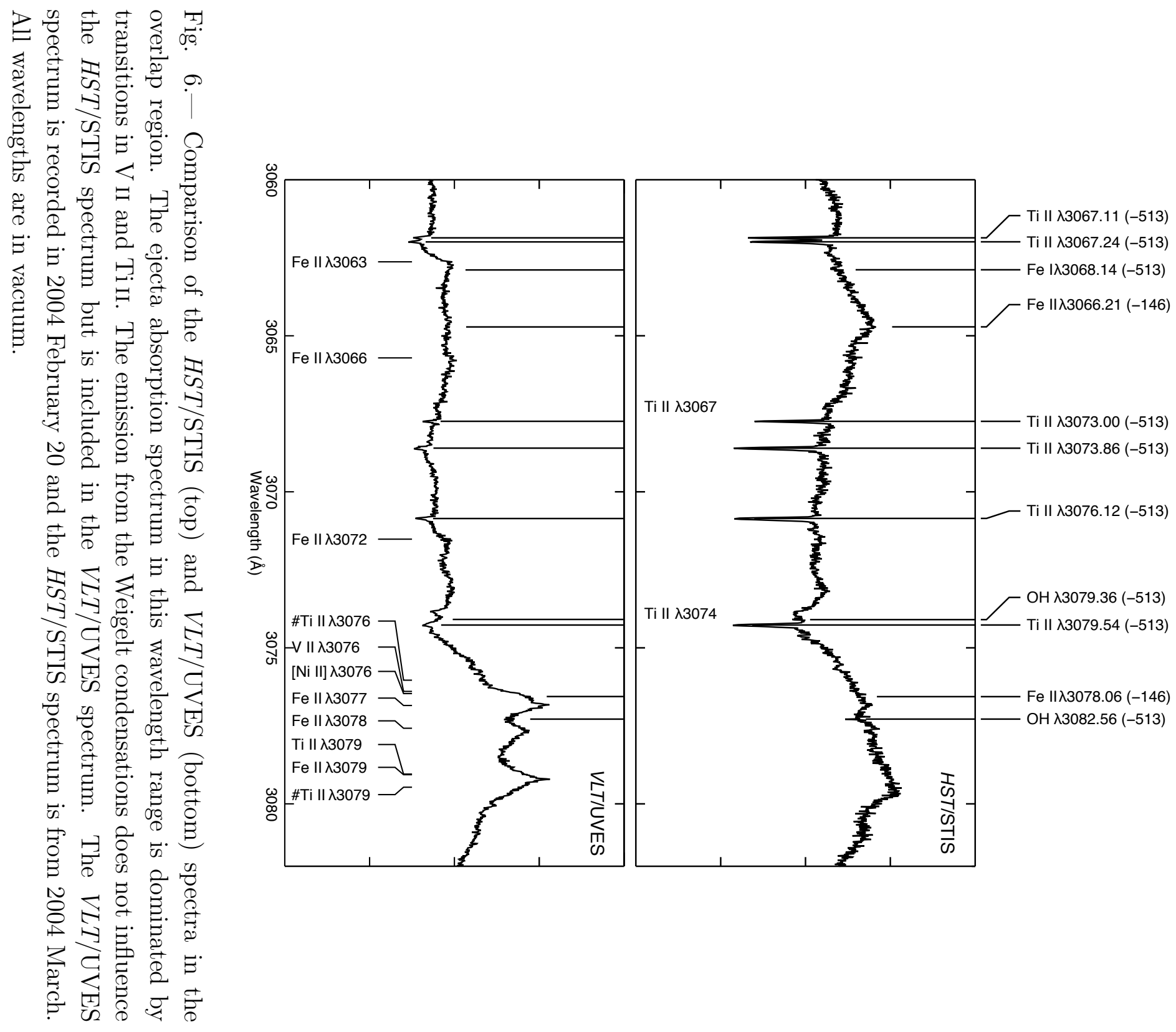




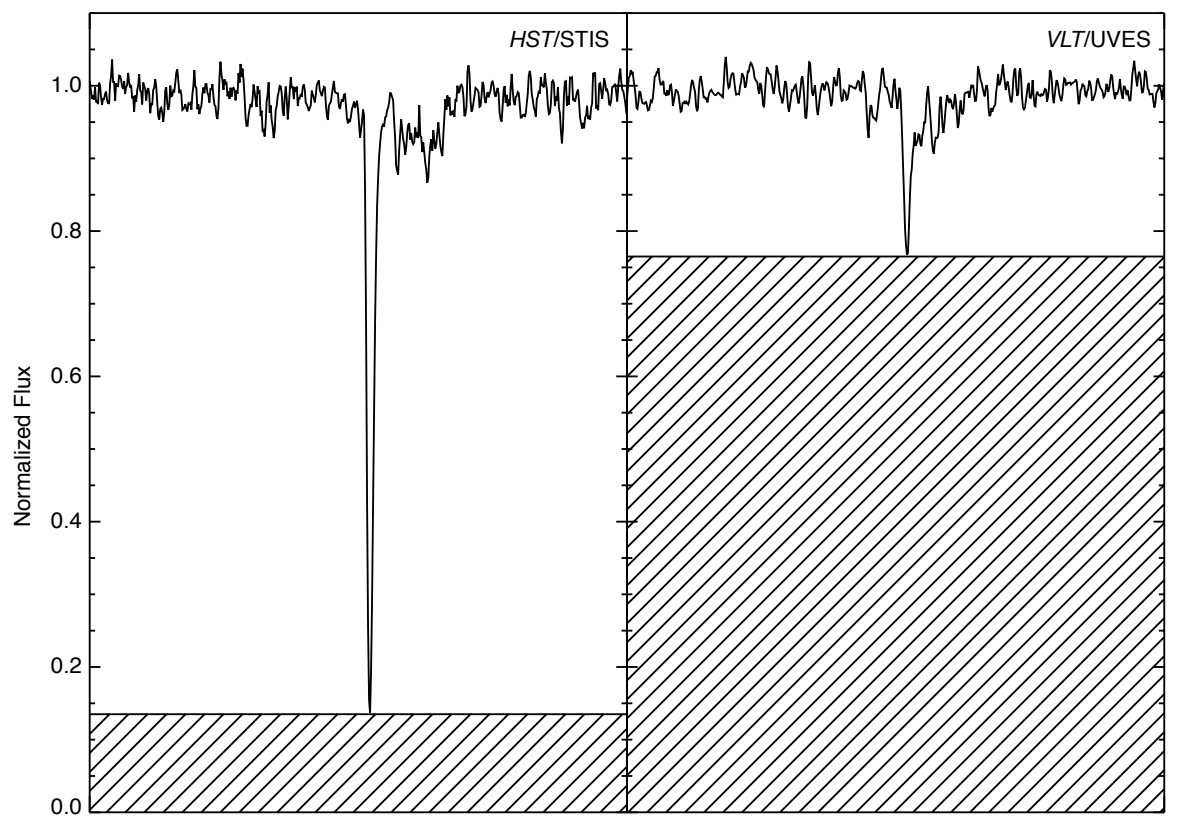

Fig. 7.- Comparison between the contribution from scattered radiation in the HST/STIS (left: spectra obtained in $2004 \mathrm{March}$ ) and the VLT/UVES (right: 2004 February 20) spectra. The wavelength region is 3080-3087 $\AA$, including the ejecta absorption lines Ti II $\lambda 3089$ shifted to $-513 \mathrm{~km} \mathrm{~s}^{-1}$. The hashed area correspond to the contribution from scattered radiation. 


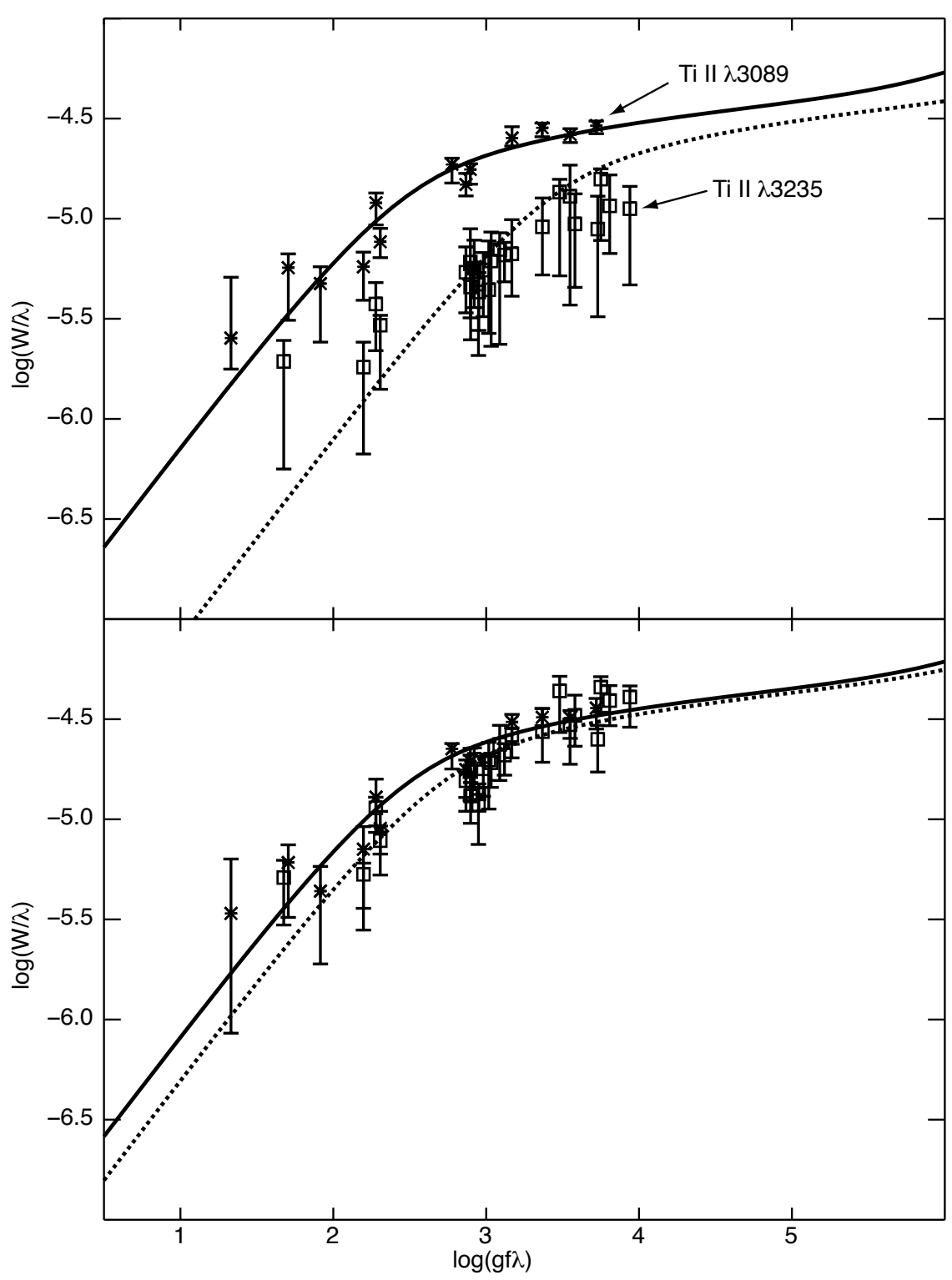

Fig. 8.- Comparison between curves-of-growth from data obtained from equivalent width measurements in the HST/STIS (solid curve) and VLT/UVES spectra (dashed curve), respectively. The HST/STIS spectrum is from 2004 March 6, while the VLT/UVES spectrum is from 2004 February 20. The upper panel show the curves-of-growth for the ground term $4 s a^{4} F$ in TiII. The column density from the STIS spectrum is nine times larger than corresponding values using the UVES spectrum. The lower panel show the curves-of-growth corrected for scattered radiation. The correction factor in the STIS spectrum is derived from the Ti II $\lambda 3089$, while Ti II $\lambda 3235$ was used for the UVES spectrum. The error bars represent the quality of the line fitting and the accuracy of the continuum placement. 


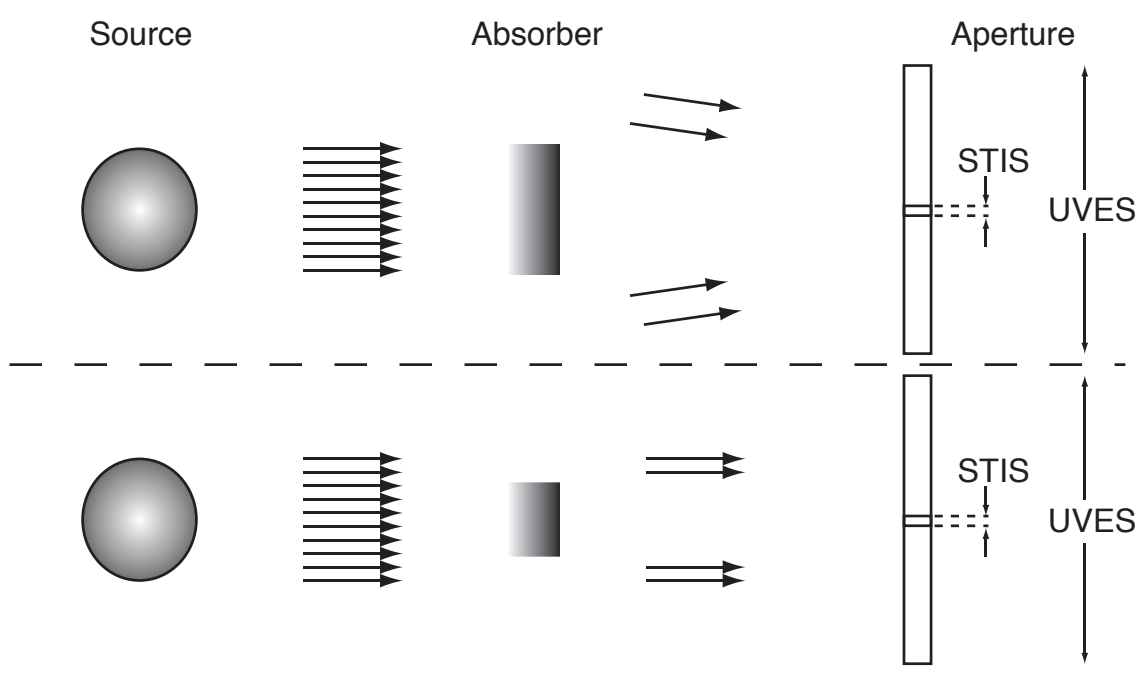

Fig. 9.- Illustration to the alternatives to explain the additional flux that enters the UVES aperture. The comparison of the UVES and STIS aperture sizes is based on the extracted parts for the spectra. In the blue spectral region the extracted part of the UVES aperture is $12 \times 0$ '” $25=33^{\prime \prime} .00$ and $7 \times 00^{\prime \prime} 0145=0$ ' 1015 for STIS. Top: The absorber, the Homunculus covers the radiation source and the additional flux contribution that enters the UVES aperture comes from scattered star light in the Homunculus itself. Bottom: The Homunculus does not completely cover the source, and the full continuum is not absorbed. For the latter to be feasible the clumpiness of the Homunculus must be significant. 


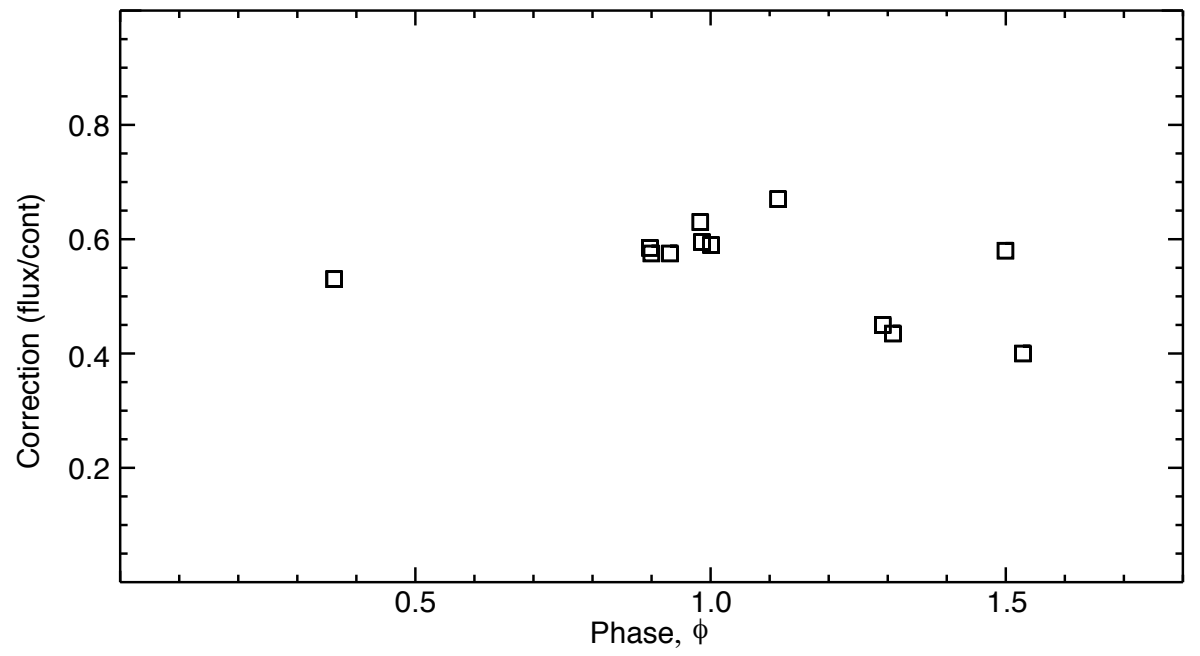

Fig. 10.- Phase dependance of the scattered radiation contributing to the continuum. The correction factor is calculated with Ti II $\lambda 3235$. The star was not centered in the aperture during the 2006 April 9 observation $(\phi=1.500)$. Consequently, the measured contribution from scatted radiation is uncertain. The phase is relative to X-ray minimum 1997.9604,

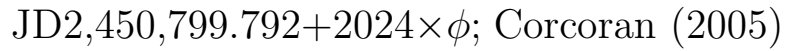




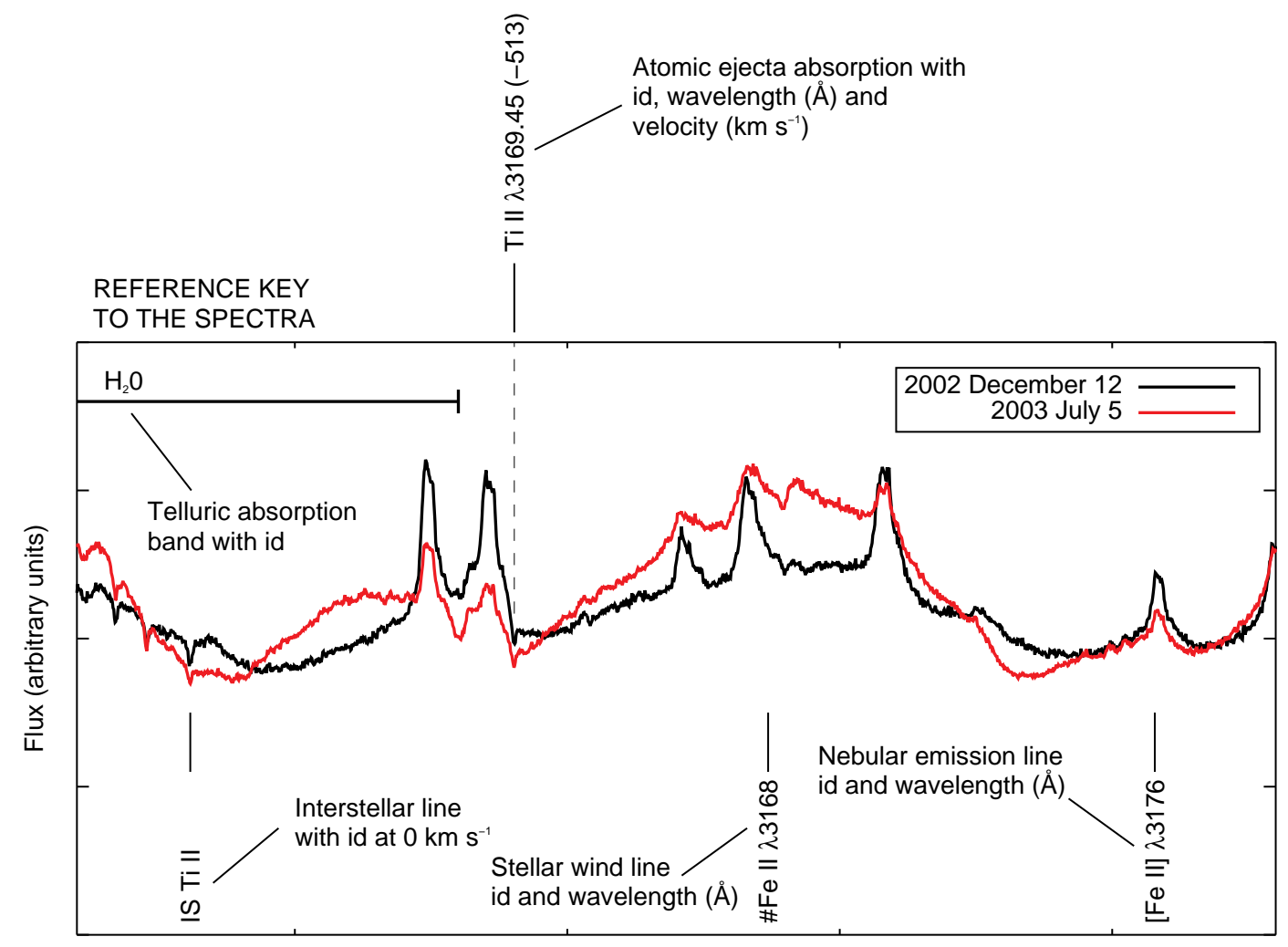

Wavelength $(\AA)$

Fig. 11. - Reference key to all online only spectra.

FIG. SET 11. - The VLT/UVES spectra. [See the electronic edition of the Supplement for Figs. 11.1-11.347] 


\section{REFERENCES}

Black, J. H., Weisheit, J. C., \& Laviana, E. 1972, ApJ, 177, 567

Bomans, D. J., Weis, K., Stahl, O., Davidson, K., Humphreys, R. M., \& Gull, T. R. 2005, in Astronomical Society of the Pacific Conference Series, Vol. 332, The Fate of the Most Massive Stars, ed. R. Humphreys \& K. Stanek, 163

Chesneau, O., Min, M., Herbst, T., Waters, L. B. F. M., Hillier, D. J., Leinert, C., de Koter, A., Pascucci, I., Jaffe, W., Köhler, R., Alvarez, C., van Boekel, R., Brandner, W., Graser, U., Lagrange, A. M., Lenzen, R., Morel, S., \& Schöller, M. 2005, A\&A, 435, 1043

Conti, P. S. 1984, in IAU Symposium, Vol. 105, Observational Tests of the Stellar Evolution Theory, ed. A. Maeder \& A. Renzini, 233

Corcoran, M. F. 2005, AJ, 129, 2018

Corcoran, M. F., Ishibashi, K., Swank, J. H., \& Petre, R. 2001, ApJ, 547, 1034

Damineli, A. 1996, ApJL, 460, L49

Damineli, A., Hillier, D. J., Corcoran, M. F., Stahl, O., Levenhagen, R. S., Leister, N. V., Groh, J. H., Teodoro, M., Albacete Colombo, J. F., Gonzalez, F., Arias, J., Levato, H., Grosso, M., Morrell, N., Gamen, R., Wallerstein, G., \& Niemela, V. 2008, MNRAS, 384,1649

Davidson, K., Dufour, R. J., Walborn, N. R., \& Gull, T. R. 1986, ApJ, 305, 867

Davidson, K., Ebbets, D., Weigelt, G., Humphreys, R. M., Hajian, A. R., Walborn, N. R., \& Rosa, M. 1995, AJ, 109, 1784

Davidson, K., Smith, N., Gull, T. R., Ishibashi, K., \& Hillier, D. J. 2001, AJ, 121, 1569

Dufour, R. J., Glover, T. W., Hester, J. J., Curie, D. G., van Orsow, D., \& Walter, D. K. 1997, in Astronomical Society of the Pacific Conference Series, Vol. 120, Luminous Blue Variables: Massive Stars in Transition, ed. A. Nota \& H. Lamers, 255

Ferland, G. J., Korista, K. T., Verner, D. A., Ferguson, J. W., Kingdon, J. B., \& Verner, E. M. 1998, PASP, 110, 761

Fuhr, J. R., Martin, G. A., \& Wiese, W. L. 1988, Atomic transition probabilities. Scandium through Manganese, Vol. 17, Suppl 4 
Gal-Yam, A., Leonard, D. C., Fox, D. B., Cenko, S. B., Soderberg, A. M., Moon, D.-S., Sand, D. J., Li, W., Filippenko, A. V., Aldering, G., \& Copin, Y. 2007, ApJ, 656, 372

Garstang, R. H. 1962, MNRAS, 124, 321

Gull, T. R., Kober, G. V., \& Nielsen, K. E. 2006, ApJS, 163, 173

Gull, T. R., Vieira, G., Bruhweiler, F., Nielsen, K. E., Verner, E., \& Danks, A. 2005, ApJ, 620,442

Hartman, H., Gull, T., Johansson, S., Smith, N., \& HST Eta Carinae Treasury Project Team. 2004, A\&A, 419, 215

Humphreys, R. M. \& Davidson, K. 1994, PASP, 106, 1025

Iping, R. C., Sonneborn, G., Gull, T. R., Massa, D. L., \& Hillier, D. J. 2005, ApJ, 633, L37

Ishibashi, K., Corcoran, M. F., Davidson, K., Swank, J. H., Petre, R., Drake, S. A., Damineli, A., \& White, S. 1999, ApJ, 524, 983

Ishibashi, K., Gull, T. R., Davidson, K., Smith, N., Lanz, T., Lindler, D., Feggans, K., Verner, E., Woodgate, B. E., Kimble, R. A., Bowers, C. W., Kraemer, S., Heap, S. R., Danks, A. C., Maran, S. P., Joseph, C. L., Kaiser, M. E., Linsky, J. L., Roesler, F., \& Weistrop, D. 2003, AJ, 125, 3222

Johansson, S. \& Letokhov, V. S. 2003, A\&A, 412, 771

Kraemer, S. B., Crenshaw, D. M., George, I. M., Netzer, H., Turner, T. J., \& Gabel, J. R. 2002, ApJ, 577, 98

Kurucz, R. L. 1988, in Trans. IAU (Kluwer: Dordrecht), ed. M. McNally, Vol. 20B (Kluwer Academic Publishers), 168-172

Lindler, D. 1999, CALSTIS Reference Guide http://hires.gsfc.nasa.gov/stis/software

Martin, J. C., Davidson, K., Humphreys, R. M., Hillier, D. J., \& Ishibashi, K. 2006, ApJ

Meynet, G. \& Maeder, A. 2000, A\&A, 361, 101

-. 2003, A\&A, 404, 975

Morse, J. A., Kellogg, J. R., Bally, J., Davidson, K., Balick, B., \& Ebbets, D. 2001, ApJ, 548, L207 
Nielsen, K. E., Corcoran, M. F., Gull, T. R., Hillier, D. J., Hamaguchi, K., Ivarsson, S., \& Lindler, D. J. 2007a, ApJ, 660, 669

Nielsen, K. E., Gull, T. R., \& Vieira Kober, G. 2005, ApJS, 157, 138

Nielsen, K. E., Ivarsson, S., \& Gull, T. R. 2007b, ApJs, 168, 289

Nilsson, H., Ljung, G., Lundberg, H., \& Nielsen, K. E. 2006, A\&A, 445, 1165

Pickering, J. C., Thorne, A. P., \& Perez, R. 2001, ApJs, 132, 403

Pittard, J. M. \& Corcoran, M. F. 2002, A\&A, 383, 636

Prochaska, J. X., Chen, H.-W., \& Bloom, J. S. 2006, ApJ, 648, 95

Raassen, A. J. J., Pickering, J. C., \& Uylings, P. H. M. 1998, A\&AS, 130, 541

Raassen, A. J. J. \& Uylings, P. H. M. 1998, A\&A, 340, 300

Smith, N. 2002, MNRAS, 337, 1252

-. 2006, ApJ, 644, 1151

Smith, N., Davidson, K., Gull, T. R., Ishibashi, K., \& Hillier, D. J. 2003a, ApJ, 586, 432

Smith, N., Gehrz, R. D., Hinz, P. M., Hoffmann, W. F., Hora, J. L., Mamajek, E. E., \& Meyer, M. R. 2003b, AJ, 125, 1458

Smith, N., Li, W., Foley, R. J., Wheeler, J. C., Pooley, D., Chornock, R., Filippenko, A. V., Silverman, J. M., Quimby, R., Bloom, J. S., \& Hansen, C. 2007, ApJ, 666, 1116

Smith, N. \& Morse, J. A. 2004, ApJ, 605, 854

Smith, N., Morse, J. A., Gull, T. R., Hillier, D. J., Gehrz, R. D., Walborn, N. R., Bautista, M., Collins, N. R., Corcoran, M. F., Damineli, A., Hamann, F., Hartman, H., Johansson, S., Stahl, O., \& Weis, K. 2004, ApJ, 605, 405

Smith, N. \& Owocki, S. P. 2006, ApJL, 645, L45

Stahl, O., Weis, K., Bomans, D. J., Davidson, K., Gull, T. R., \& Humphreys, R. M. 2005, A\&A, 435, 303

Steiner, J. E. \& Damineli, A. 2004, ApJ, 612, L133 
Valenti, J., Busko, I., Quijano, J. K., Lindler, D., \& Bowers, C. W. 2002, in The 2002 HST Calibration Workshop : Hubble after the Installation of the ACS and the NICMOS Cooling System, Proceedings of a Workshop held at the Space Telescope Science Institute, Baltimore, Maryland, October 17 and 18, 2002. Edited by Santiago Arribas, Anton Koekemoer, and Brad Whitmore. Baltimore, MD: Space Telescope Science Institute, 2002., 210

van Boekel, R., Kervella, P., Schöller, M., Herbst, T., Brandner, W., de Koter, A., Waters, L. B. F. M., Hillier, D. J., Paresce, F., Lenzen, R., \& Lagrange, A.-M. 2003, A\&A, 410, L37

van Genderen, A. M., Sterken, C., Allen, W. H., \& Liller, W. 2003, A\&A, 412, L25

Verner, E., Bruhweiler, F., Nielsen, K. E., Gull, T. R., Vieira Kober, G., \& Corcoran, M. 2005a, ApJ, 629, 1034

Verner, E. M., Bruhweiler, F., \& Gull, T. R. 2005b, ApJ, 624, 973

Vreeswijk, P. M., Ledoux, C., Smette, A., Ellison, S. L., Jaunsen, A. O., Andersen, M. I., Fruchter, A. S., Fynbo, J. P. U., Hjorth, J., Kaufer, A., Møller, P., Petitjean, P., Savaglio, S., \& Wijers, R. A. M. J. 2007, A\&A, 468, 83

Weigelt, G. \& Ebersberger, J. 1986, A\&A, 163, L5

Weigelt, G., Kraus, S., Driebe, T., Petrov, R. G., Hofmann, K.-H., Millour, F., Chesneau, O., Schertl, D., Malbet, F., Hillier, J. D., Gull, T., Davidson, K., Domiciano de Souza, A., Antonelli, P., Beckmann, U., Bresson, Y., Chelli, A., Dugué, M., Duvert, G., Gennari, S., Glück, L., Kern, P., Lagarde, S., Le Coarer, E., Lisi, F., Perraut, K., Puget, P., Rantakyrö, F., Robbe-Dubois, S., Roussel, A., Tatulli, E., Zins, G., Accardo, M., Acke, B., Agabi, K., Altariba, E., Arezki, B., Aristidi, E., Baffa, C., Behrend, J., Blöcker, T., Bonhomme, S., Busoni, S., Cassaing, F., Clausse, J.-M., Colin, J., Connot, C., Delboulbé, A., Feautrier, P., Ferruzzi, D., Forveille, T., Fossat, E., Foy, R., Fraix-Burnet, D., Gallardo, A., Giani, E., Gil, C., Glentzlin, A., Heiden, M., Heininger, M., Hernandez Utrera, O., Kamm, D., Kiekebusch, M., Le Contel, D., Le Contel, J.-M., Lesourd, T., Lopez, B., Lopez, M., Magnard, Y., Marconi, A., Mars, G., Martinot-Lagarde, G., Mathias, P., Mège, P., Monin, J.-L., Mouillet, D., Mourard, D., Nussbaum, E., Ohnaka, K., Pacheco, J., Perrier, C., Rabbia, Y., Rebattu, S., Reynaud, F., Richichi, A., Robini, A., Sacchettini, M., Schöller, M., Solscheid, W., Spang, A., Stee, P., Stefanini, P., Tallon, M., Tallon-Bosc, I., Tasso, D., Testi, L., Vakili, F., von der Lühe, O., Valtier, J.-C., Vannier, M., Ventura, N., Weis, K., \& Wittkowski, M. 2007, A\&A, 464, 87 
Weis, K., Bomans, D. J., Stahl, O., Davidson, K., Humphreys, R. M., \& Gull, T. R. 2005, in Astronomical Society of the Pacific Conference Series, Vol. 332, The Fate of the Most Massive Stars, ed. R. Humphreys \& K. Stanek, 160

Weis, K., Corcoran, M. F., Bomans, D. J., \& Davidson, K. 2004, A\&A, 415, 595

Weis, K. \& Duschl, W. J. 1999, in Astronomical Society of the Pacific Conference Series, Vol. 179, Eta Carinae at The Millennium, ed. J. A. Morse, R. M. Humphreys, \& A. Damineli, 155

Whitelock, P. A., Feast, M. W., Marang, F., \& Breedt, E. 2004, MNRAS, 352, 447

Zanella, R., Wolf, B., \& Stahl, O. 1984, A\&A, 137, 79

Zethson, T. 2001, PhD thesis, Lund University

Zethson, T., Gull, T. R., Hartman, H., Johansson, S., Davidson, K., \& Ishibashi, K. 2001, AJ, 122,322 